\title{
The Formation of Ultra Diffuse Galaxies in Cored Dark Matter Halos Through Tidal Stripping and Heating
}

\author{
Timothy Carleton, ${ }^{1,3 \star}$ Raphaël Errani, ${ }^{2}$ \\ Michael Cooper, ${ }^{1}$ Manoj Kaplinghat, ${ }^{1}$ Jorge Peñarrubia, ${ }^{2}$ Yicheng Guo ${ }^{3}$ \\ ${ }^{1}$ Center for Cosmology, Department of Physics and Astronomy, 4129 Reines Hall, University of California, Irvine, CA 92697, USA \\ ${ }^{2}$ Institute for Astronomy, University of Edinburgh, Royal Observatory, Blackford Hill, Edinburgh EH9 3HJ, UK \\ ${ }^{3}$ Department of Physics and Astronomy, 223 Physics Building, University of Missouri, Columbia, MO 65211, USA
}

6 February 2019

\begin{abstract}
We propose that the Ultra-Diffuse Galaxy (UDG) population represents a set of satellite galaxies born in $\sim 10^{10}-10^{11} \mathrm{M}_{\odot}$ halos, similar to field dwarfs, which suffer a dramatic reduction in surface brightness due to tidal stripping and heating. This scenario is observationally motivated by the radial alignment of UDGs in Coma as well as the significant dependence of UDG abundance on cluster mass. As a test of this formation scenario, we apply a semi-analytic model describing the change in stellar mass and half-light radius of dwarf satellites, occupying either cored or cuspy halos, to cluster subhalos in the Illustris-dark simulation. Key to this model are results from simulations which indicate that galaxies in cored dark-matter halos expand significantly in response to tidal stripping and heating, whereas galaxies in cuspy halos experience limited size evolution. Our analysis indicates that a population of tidallystripped dwarf galaxies, residing in cored halos (like those hosting low-surface brightness field dwarfs), is able to reproduce the observed sizes and stellar masses of UDGs in clusters remarkably well.
\end{abstract}

Key words: galaxies: formation, evolution, dwarf, halos, clusters, kinematics and dynamics

\section{INTRODUCTION}

The recent identification of a large population of ultra-diffuse galaxies (UDGs) in the Coma cluster (van Dokkum et al. 2015a,b) has led to a renewed interest in low surface brightness galaxies. Since the original identification of 47 UDGs in Coma, hundreds more have been identified in the Coma, Perseus, Fornax, and Virgo clusters (Koda et al. 2015; Mihos et al. 2015; Muñoz et al. 2015; Yagi et al. 2016; Wittmann et al. 2017; Venhola et al. 2017; Ordenes-Briceño et al. 2018; Eigenthaler et al. 2018) as well as in higher-redshift clusters (Lee et al. 2017; Janssens et al. 2017) and lower-mass groups (Martin et al. 2016; Merritt et al. 2016; Smith Castelli et al. 2016; Toloba et al. 2016; Román \& Trujillo 2017b; Shi et al. 2017; Spekkens \& Karunakaran 2018; Müller et al. 2018). These cluster UDGs are characterized by old stellar populations, stellar masses of $10^{7}-10^{8} \mathrm{M}_{\odot}$, and exponential light profiles similar to typical low-mass dwarf ellipticals, but with half-light radii $>1.5 \mathrm{kpc}$ and central surface brightnesses $>24 \mathrm{mag} \mathrm{arcsec}^{-2}$. Although UDGs represent a minority of the overall dwarf galaxy pop-

^ e-mail: carletont@missouri.edu ulation, the peculiar nature of these systems provides an excellent testbed for our understanding of galaxy evolution.

The unusual nature of these systems has sparked a number of theories regarding their formation. In particular, the dark matter content of UDGs has been subject to extensive debate, with some investigations, using both stellar velocity dispersions (van Dokkum et al. 2016) and HI dynamics (Trujillo et al. 2017), arriving at the conclusion that UDGs are extremely dark matter dominated in their central regions, in agreement with globular cluster-based mass determinations (Peng \& Lim 2016; Beasley et al. 2016; van Dokkum et al. 2017; Toloba et al. 2018). Other studies, also using globular cluster velocity dispersions (van Dokkum et al. 2018), as well as globular cluster colors and abundances (Amorisco et al. 2016; Beasley \& Trujillo 2016; Amorisco et al. 2018) have found that the mass-to-light ratio of UDGs is consistent with that of similarly massive dwarfs (however, Laporte et al. (2018) and Martin et al. (2018) have cautioned against the use a limited number of globular clusters to estimate the dynamical mass of UDGs, citing significant systematic uncertainties). This latter conclusion is consistent with weak gravitational lensing limits (Sifón et al. 2018) and observations of the distribution of UDGs in groups and clusters (Román \& Trujillo 2017a). Additional evidence that UDGs live in dwarf-scale 
halos derives from the abundance of UDGs in clusters: there simply are not enough Milky Way-mass halos in clusters to host $90 \%$ of the UDG population (Amorisco 2018).

Prompted by the observation of a large internal velocity dispersion for a UDG in Coma, van Dokkum et al. (2016) hypothesized a formation scenario in which UDGs are born within Milky Way-like dark matter halos but fail to form most of their stars due to extreme feedback (see also Agertz \& Kravtsov 2016). Similarly, it has been suggested that UDGs are formed when strong feedback expands dwarf galaxies, which retain their large sizes as they fall into a cluster (Di Cintio et al. 2017; Chan et al. 2018). The feedback required in this scenario finds support in recent simulations in which dwarf galaxies experience cycles of expansion and contraction due to large stellar feedback effects (Pontzen \& Governato 2014). These simulations with extreme feedback, however, overpredict the sizes of isolated dwarf galaxies (Lange et al. 2015) and are not able to reproduce the relative abundance of cluster vs. field UDGs. Alternatively, it has been suggested that UDGs represent galaxies living in halos in the tail of the spin distribution (Amorisco \& Loeb 2016; Leisman et al. 2017). While this scenario does a better job of reproducing the observed size distribution, it predicts that cluster UDGs should be less abundant and biased towards less-diffuse systems compared with UDGs in the field, contrary to observations (Román \& Trujillo 2017b; Jones et al. 2017).

Clues to the UDG formation mechanism have come as more complete observations of the environments hosting UDGs have been conducted. Although UDGs are observed outside cluster environments (Leisman et al. 2017; Román \& Trujillo 2017b; Williams et al. 2016), cluster UDGs are both more diffuse and relatively more abundant than isolated UDGs. A systematic survey of UDGs in clusters from van der Burg et al. (2017) found that the relative abundance of UDGs increases with increasing cluster mass, consistent with an increase in the mass fraction of UDGs from $\sim 0.1 \%$ in the field (Leisman et al. 2017; Jones et al. 2017) to $\sim 3 \%$ in clusters (van der Burg et al. 2016; Yagi et al. 2016). Additionally, Román \& Trujillo (2017b) found that UDGs within $300 \mathrm{kpc}$ of the Hickson Compact Groups HCG07, HCG25, and HCG98 have lower central surface brightnesses compared with UDGs at farther distances from the group center. Furthermore, there is a significant radial alignment observed in Coma UDGs (Yagi et al. 2016; but see Venhola et al. 2017; Eigenthaler et al. 2018), suggesting that the shapes of these systems have been affected by tidal interactions. Lastly, Burkert (2017) show that the axis ratios of Coma UDGs are more consistent with elongated structures than puffed-up disks. These distinctions strongly suggest that environmental mechanisms are at play in the formation and evolution of UDGs. In fact, 'galaxy harassment,' tidal stripping, and ram-pressure stripping have recently been suggested as possible UDG formation mechanisms (Safarzadeh \& Scannapieco 2017; Conselice 2018; Ogiya 2018; Bennet et al. 2018).

Within the Local Group, studies have shown that satellite galaxies are significantly influenced by the tidal effects of a massive host galaxy. For example, Peñarrubia et al. (2010) find that satellites orbiting the Milky Way and Andromeda can lose over 99\% of their mass during the course of a few orbits due to tidal stripping. Importantly, they show that tidal stripping affects cored and cuspy halos differently: cored halos endure a greater degree of stripping than cuspy halos, and cuspy halos experience stripping primarily in the halo outskirts, whereas tidal stripping of cored halos occurs more evenly throughout the halo. Following this work, Errani et al. (2015) investigated the effect of tidal stripping on satellite galaxies. While the stellar population of satellites in both cored and cuspy halos experiences expansion as a consequence of tidal heating, as well as stellar stripping, satellites in cored halos are able to grow in size significantly before much stellar stripping occurs. After a cuspy halo loses $50 \%$ of its mass within the half-light radius due to tides, the satellite residing in the halo expands by $20 \%$, whereas a satellite hosted by a similarly stripped cored halo expands by over a factor of 2. Given that there is evidence that dwarf galaxies with stellar masses around $10^{8} \mathrm{M}_{\odot}$ live in cored dark matter halos (e.g. Kuzio de Naray et al. 2008; Oh et al. 2011), stellar expansion as a result of tidal heating appears to be a viable UDG formation scenario.

Motivated by the environmental dependence of UDG formation as well as the predicted expansion of dwarf galaxies through tidal heating, we present a scenario in which UDGs are formed through tidal heating as dwarf galaxies orbit within a cluster. Using subhalos identified in the Illustris-dark simulation, we illustrate that the observed distribution of UDG properties are consistent with this scenario. In Section 2, we describe the selection of cluster subhalos from Illustris-dark and the association of dwarf galaxies with subhalos at infall. In Section 3 and 4, we outline our procedure for modeling the effect of tidal stripping and compare the properties of UDGs produced via tidal stripping with the observed cluster UDGs, before summarizing our conclusions in Section 5.

\section{IDENTIFICATION OF CLUSTER SUBHALOS}

In order to explore the effect that tidal stripping has on the properties of dwarf galaxies, we utilize data from the Illustris-dark Simulation (Vogelsberger et al. 2014a; Nelson et al. 2015). Illustrisdark is a cosmological, dark matter-only simulation of $1820^{3}$ particles, each with a mass of $5.32 \times 10^{6} h^{-1} \mathrm{M}_{\odot}$, in a $75 h^{-1} \mathrm{Mpc}$ box, simulated using a Plummer-equivalent force softening length of 1.0 comoving $h^{-1} \mathrm{kpc}$. Illustris-dark was run with the following cosmological parameters: $h=0.704, \Omega_{\mathrm{M}}=0.2726, \Omega_{\Lambda}=0.7274$, $n_{s}=0.963$, and $\sigma_{8}=0.809$. Halos with masses down to $10^{8} \mathrm{M}_{\odot}$ are identified using the Subfind algorithm (Springel et al. 2001), and the merger trees are generated using the Sublink algorithm (Rodriguez-Gomez et al. 2015), with halo properties output for 135 time-steps logarithmically spaced in scale factor. As we discuss in Section 3, the only subhalo properties we utilize directly from the simulations are the subhalo masses at infall and the subhalo orbits. These properties are accurate to a high level $(<10 \mathrm{kpc}$ in position and $<10 \mathrm{~km} \mathrm{~s}^{-1}$ in velocity; Behroozi et al. 2013a).

Given that halos in cluster environments are most likely to experience significant tidal stripping, we restrict our analysis to subhalos within $R_{200}$ of a cluster with $M_{200}>10^{14} \mathrm{M}_{\odot}$ at $z=0$. Here, and for the remainder of this work, $R_{\Delta}$ represents the radius within which the mean density of a halo is $\Delta$ times the critical density and $M_{\Delta}$ is the bound mass within $R_{\Delta}$. Subhalo virial masses $\left(M_{\mathrm{vir}}\right)$ are defined according to the Bryan \& Norman (1998) redshiftdependent criteria, which corresponds to $\Delta=97$ at $z=0$ for the Illustris-dark cosmology. Each subhalo is assigned a galaxy with a stellar mass based on the maximum value of $M_{\mathrm{vir}}$ before infall given by the stellar mass-halo mass relation for cluster environments from Kravtsov et al. (2018), with 0.2 dex normally-distributed scatter (Behroozi et al. 2013b).

\subsection{Galaxy Sizes at Infall}

In addition to the stellar mass determined from abundance matching, each galaxy is assigned a half-light radius at infall based on 
its stellar mass, following the observed size-mass relation from the GAMA survey (Driver et al. 2011; Lange et al. 2015; Liske et al. 2015). Given that low-mass dwarf galaxies are quenched rapidly after falling into a massive host halo (e.g. Fillingham et al. 2015, 2016), we use the size-mass relation for red galaxies as our initial condition:

$$
r_{e}=0.17\left(\frac{M_{*}}{\mathrm{M}_{\odot}}\right)^{0.1}\left(1+\frac{M_{*}}{2.31 \times 10^{10} \mathrm{M}_{\odot}}\right)^{0.65},
$$

where $M_{*}$ is the stellar mass assigned to the subhalo at infall. The intrinsic scatter in $\log \left(r_{e}\right)$ at a given mass is taken to be $0.1 \mathrm{dex}$, following measurements of the size-mass relation in CANDELS (Grogin et al. 2011; Koekemoer et al. 2011; van der Wel et al. 2014). While observations suggest significant size evolution of galaxy sizes with redshift among massive galaxies (van der Wel et al. 2014), the evolution of low-mass galaxies with $z$ is less clear. In contrast with observations of a significant decrease in galaxy sizes with $z$ among massive galaxies, hydrodynamic simulations find that dwarf galaxy sizes are primarily driven by cycles of star-formation and supernova feedback, and do not strongly evolve with $z$ (Chan et al. 2018; El-Badry et al. 2016). Furthermore, if we include the redshift-dependence of $r_{e}$ for massive galaxies from van der Wel et al. (2014) in our model, the $z=0$ dwarf galaxy population does not lie along the observed size-mass distribution of quiescent systems, so we do not include any redshift dependence in our starting size-mass relation.

The applicability of this starting condition remains the largest source of uncertainty in our analysis. Understanding the processes involved in the transformation from star-forming dwarf-irregular galaxies (following the size-mass relation for blue systems), to quiescent dwarf-ellipticals (following the size-mass relation for red galaxies) is an active area of research (e.g. Wheeler et al. 2017; Kazantzidis et al. 2017; Fattahi et al. 2018). As quenching and tidal heating take place on similar timescales in this mass range ( $\sim 1 \mathrm{Gyr})$, and the vast majority of quiescent galaxies with $M_{*}=10^{8} \mathrm{M}_{\odot}$ live in dense environments (Geha et al. 2012), it is very difficult to disentangle the processes of tidal heating and quenching that drive the size evolution of these dwarfs.

Regardless, there is evidence that most $M_{*} \sim 10^{8} \mathrm{M}_{\odot}$ cluster dwarfs obey Eqn. 1 before tidal stripping occurs. Firstly, most cluster dwarfs (including UDGs; Roediger et al. 2017; Eigenthaler et al. 2018; Yagi et al. 2016) in this mass range are quenched, suggesting that any internal processes altering their sizes should have occurred at some point before we observe them. Similarly, observations indicate that $\sim 70 \%$ of UDGs are nucleated (Yagi et al. 2016), implying that they were smaller systems with a more significant bulge before expanding. Additionally, Eqn. 1, in contrast with the sizemass relation for blue systems, produces a substantial population of $M_{*} \sim 10^{8} \mathrm{M}_{\odot}$ dwarf-ellipticals with small $\left(r_{e}<1 \mathrm{kpc}\right)$ sizes, in agreement with the observed size-mass relation for cluster dwarfs. In particular, the size distribution of $5 \times 10^{7}-2 \times 10^{8} \mathrm{M}_{\odot}$ dwarfs produced through this analysis following Eqn. 1 generally agrees with the size distribution of dwarfs of similar mass identified in the Next Generation Fornax Survey (Muñoz et al. 2015). Lastly, if the size-mass relation for blue galaxies is used as the initial condition for this analysis, the size distribution is flatter than observed (see Sec. 4.1).

However, there is also evidence that some systems may follow the size-mass relation for blue galaxies before they are stripped. If all dwarfs follow the quiescent size-mass relation before stripping, it is difficult to fully reproduce either the large velocity dispersions observed in some UDGs (Beasley et al. 2016; van Dokkum

\begin{tabular}{lcccrrr}
\hline$\gamma$ & $r_{e} / r_{\max }$ & & $M_{*}$ & $r_{e}$ & $V_{\max }$ & $r_{\max }$ \\
\hline 1 (cusp) & 0.05 & $\alpha$ & 1.87 & 0.47 & 0.40 & -0.30 \\
1 & 0.05 & $\beta$ & 1.87 & 0.41 & 0.30 & 0.40 \\
1 & 0.05 & $\log _{10}\left(x_{s}\right)$ & -2.64 & -2.64 & 0 & 0 \\
1 & 0.1 & $\alpha$ & 1.80 & 0.50 & 0.40 & -0.30 \\
1 & 0.1 & $\beta$ & 1.80 & 0.42 & 0.30 & 0.40 \\
1 & 0.1 & $\log _{10}\left(x_{s}\right)$ & -2.08 & -2.08 & 0 & 0 \\
\hline 0 (core) & 0.05 & $\alpha$ & 2.83 & -0.25 & 0.40 & -1.30 \\
0 & 0.05 & $\beta$ & 2.83 & -0.25 & 0.37 & 0.05 \\
0 & 0.05 & $\log _{10}\left(x_{s}\right)$ & -3.12 & 0 & 0 & 0 \\
0 & 0.1 & $\alpha$ & 0.330 & 2.05 & 0.40 & -1.30 \\
0 & 0.1 & $\beta$ & 0.140 & 2.05 & 0.37 & 0.05 \\
0 & 0.1 & $\log _{10}\left(x_{s}\right)$ & 0 & 0 & 0 & 0 \\
\hline
\end{tabular}

Table 1. The $\alpha, \beta$, and $x_{s}$ parameters, for a given $\gamma, r_{e} / r_{\max }$, utilized in Equation 2 to model the changes in stellar mass, half-light radius, halo $V_{\max }$, and halo $r_{\max }$ as a consequence of tidal stripping. Note that the $r_{e}$ and $M_{*}$ tracks, generated among subhalos in the Aquarius-A2 simulations, take the ratio of the final mass within $r_{\max }$ to the initial mass within $r_{\max }$ as $x$ in Equation 2, whereas the $r_{\max }$ and $V_{\max }$ tracks, generated by simulating multiple subhalo orbits within a Galactic potential, take ratio of the initial mass within the tidal radius to the final mass within the tidal radius as $x$ (see Sec. 3).

et al. 2016, see Sec. 4.3) or the abundance of extremely extended (>4.5 kpc) UDGs (see Sec. 4.1). If some UDG progenitors lie closer to the size-mass relation for blue systems from the GAMA survey, such extreme UDGs are produced in our model. Furthermore, the quenching time associated with more massive dwarfs is significantly longer than the orbital time of cluster subhalos (Wheeler et al. 2014), suggesting that they continue to form stars as tidal stripping and heating occur.

The true relationship between the size and mass of dwarfs before they are stripped is likely in-between the star-forming and quiescent relations, particularly for systems with high $M_{*}$ at infall (that may take longer to quench) or systems that were accreted at high$z$ (when galaxies may have been smaller in the field and clusters may have been less effective at quenching infalling systems). Ultimately, we adopt the $z=0$ size-mass relation for quiescent systems as a straightforward, conservative estimate of the size distribution of cluster dwarfs before they are stripped, as it best matches the joint size distributions of cluster UDGs and dwarf-ellipticals.

\section{MODELING THE EFFECT OF TIDES ON CLUSTER SATELLITES}

In order to model the effect that tides have on the size and stellar mass of dwarf galaxies, we make use of results from Errani et al. (2015). They find that the change in stellar mass and stellar half-light radius of a tidally-stripped and heated galaxy can be parameterized as a function of the total amount of mass lost within the half-light radius and the inner slope of the dark matter density profile. To improve the applicability of this tidal parameterization to cosmological simulations, updated tidal tracks have been created using satellites drawn from the Aquarius-A2 merger tree (Springel et al. 2008) in which the stellar population is treated as a collection of collisionless tracer particles (Errani et al. 2017, 2018) and the dark-matter halo is set up initially as a Dehnen (1993) profile. These updated tracks (originally introduced in Peñarrubia et al. 2008) are parameterized as a function of the mass loss experienced 


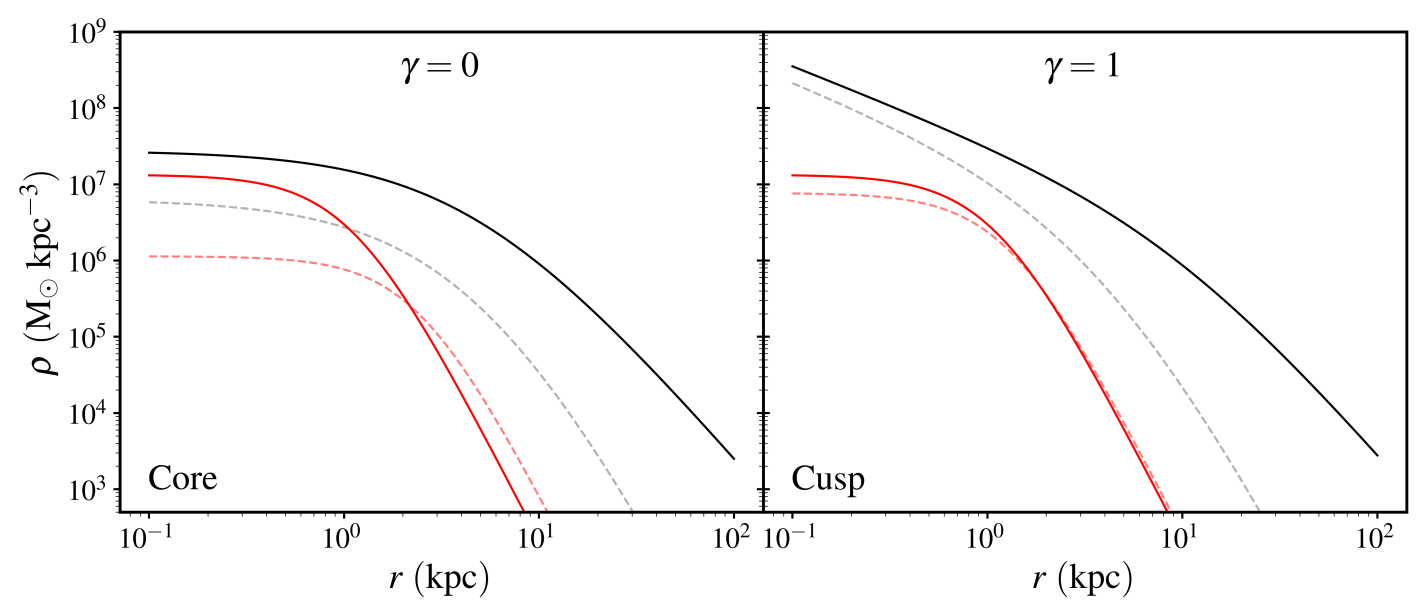

Figure 1. The effect of tidal stripping on galaxies in both cuspy (right) and cored (left) dark matter halos. The black and the red lines represent the dark matter and stellar density distributions for a representative system at infall. After the galaxy is tidally stripped, the resulting stellar and dark matter density distributions are shown as dashed light red and grey lines, respectively. At infall, the galaxy has a dark matter mass of $3.6 \times 10^{10} \mathrm{M}_{\odot}$ and stellar mass of $7.5 \times 10^{7} \mathrm{M}_{\odot}$, with a stellar half-light radius of $1.1 \mathrm{kpc}$. After three pericentric passage within $350 \mathrm{kpc}$ of a cluster with $M_{200}=2.4 \times 10^{14} \mathrm{M}_{\odot}$, the halo loses $98 \%$ of its mass. The cored and cuspy systems have similar stellar masses at $z=0\left(6.7 \times 10^{7} \mathrm{M}_{\odot}\right.$ and $6.9 \times 10^{7} \mathrm{M}_{\odot}$, respectively); however, the half-light radius of the cored system grows to $2.4 \mathrm{kpc}$, while the half-light radius of the cuspy system only grows slightly to $1.3 \mathrm{kpc}$.

within the radius corresponding to the maximum circular velocity $\left(r_{\max }\right)$. Both the change in stellar mass and stellar half-light radius are expressed using the functional form:

$$
g(x)=\frac{\left(1+x_{s}\right)^{\alpha} x^{\beta}}{\left(x+x_{s}\right)^{\alpha}},
$$

where $g(x)$ represents either $M_{*}$, final $/ M_{*}$, initial or $r_{e}$, final $/ r_{e}$, initial and $x$ is $M\left(r<r_{\max }\right)_{z=0} / M\left(r<r_{\max }\right)_{\text {infall, }}$, with $r_{\max }$ evaluated at $z=0$ and infall, respectively. As given in Table 1 , the values for $\alpha, \beta$, and $x_{s}$ are determined by fitting $g(x)$ to systems with $<99 \%$ mass loss and depend on the inner slope of the dark matter density profile, $\gamma$, as well as the ratio between $r_{e}$ and $r_{\max }$. Although a second-order dependence of the tracks on the shape of the stellar distribution is apparent, a Plummer density distribution (Plummer 1911) for the stellar profile of all satellites is assumed given the limited information regarding the stellar distributions of UDGs. Given that the $r_{e} / r_{\max }$ values within our sample span a wide range (the $5-95$ percentile range is 0.04 to 0.28 ), we logarithmically interpolate (and extrapolate when required) between the distinct mass-loss tracks to determine $g(x)$ for each halo, with the condition that $M_{* \text {, final }} / M_{*}$, initial $\leq 1$. Although this requires significant extrapolation, the tracks only weakly depend on $r_{e} / r_{\max }$ (the median absolute deviation of the interpolated/extrapolated tracks from the measured tracks due to this correction is $1 \%$ both in terms of $M_{*, \text { final }} / M_{* \text {, initial }}$ or $r_{e, \text { final }} / r_{e}$, initial $)$; the adoption of a constant $r_{e} / r_{\max }$ does not significantly impact our results.

\subsection{Mass Loss Experienced by Subhalos}

This analysis relies on an accurate measurement of the amount of mass lost as a result of tides for each subhalo. As Illustris-dark was run with cold collisionless dark matter particles, the simulation only produces cuspy halo density profiles. Thus, the mass loss experienced by halos in these simulations is insufficient to model the tidal effects experienced by cored dwarfs. Moreover, recent work by van den Bosch et al. (2016), van den Bosch (2017), and van den Bosch et al. (2018) show that up to $80 \%$ of tidal stripping observed in the Bolshoi simulation (Klypin et al. 2011) could be the result of numerical artifacts rather than physical stripping. In lieu of the mass loss as measured in Illustris-dark, we use the procedure outlined in Peñarrubia et al. (2010) to determine the change in mass and shape of dark matter halos as a result of tidal stripping. This procedure, briefly outlined below, only relies on knowledge of the subhalo orbit and the initial halo profile, which are subject to significantly less uncertainty than the measured subhalo properties at $z=0$, to determine the amount of stripping.

For each subhalo, we track its orbit from infall to the first pericentric passage. At pericenter, the amount of bound mass is calculated as the total mass within the tidal radius. Following van den Bosch et al. (2018), we calculate the tidal radius as

$$
R_{t, 1}=\left(\frac{G m_{\mathrm{sat}}\left(R_{t, 1}\right)}{\omega^{2}-\frac{d^{2} \Phi}{d r^{2}}}\right)^{1 / 3}
$$

or

$$
R_{t, 2}=D\left(\frac{m_{\mathrm{sat}}\left(R_{t, 2}\right)}{M_{\mathrm{host}}(D)}\right)
$$

where $m_{\mathrm{sat}}\left(R_{t}\right)$ is the mass of the galaxy within the tidal radius (including both the dark-matter and stellar component), $D$ is the distance between the satellite and the host cluster, $M_{\text {host }}(D)$ is the mass of the host cluster within $D$, and $\omega$ is the angular velocity of the subhalo, measured directly from the simulation. The second derivative of the host potential at pericenter, $\frac{d^{2} \Phi}{d r^{2}}$, is calculated assuming an NFW profile, using the mass and scale radius of the cluster identified in the halo catalogs. Following van den Bosch et al. (2018), we chose $R_{t, 1}$ or $R_{t, 2}$ as the tidal radius depends on the angular momentum of the orbit normalized by the angular momentum of a circular orbit with the same energy $(\eta)$. If $\eta$ is greater than 0.75 , the tidal radius is taken to be $R_{t, 1}$; otherwise we use $R_{t, 2}$.

The mass within the tidal radius at pericenter divided by the maximum virial mass of the subhalo before infall $\left(M_{\text {peak }}\right)$ is then used as input for the tidal tracks (Eqn. 2, using the $\alpha, \beta$, and $x_{s}$ values from Table 1) to determine the change in $V_{\max }$ and $r_{\max }$ of the halo. For halos that do not have a pericenter before $z=0$, the mass within the tidal radius at $z=0$ divided by $M_{\text {peak }}$ is used to determine the changes in $r_{\max }$ and $V_{\max }$. If a halo expe- 
riences $>10 \%$ mass loss, the log-slope of the outer density profile is changed from 3 to 5 to match the observed outer density profiles of tidally-stripped galaxies (Peñarrubia et al. 2008, 2009). This procedure is repeated for each pericentric passage to establish the $z=0$ profile. This analysis has been shown to reproduce the amount of mass loss in halos experiencing tidal stripping (see Appendix A of Peñarrubia et al. 2010). Among halos with more than 10000 particles at infall in the Illustris-dark simulation, the mass loss produced through this technique reproduces the simulated mass loss well (with $\left\langle\log \left[M_{z=0}\right.\right.$, model $\left.\left./ M_{z=0, \text { sim }}\right]\right\rangle=0.28$ and $\left.\sigma_{\log \left[M_{z=0, \operatorname{model}} / M_{z=0, \operatorname{sim}}\right]}=0.43\right)$.

In line with results from cosmological simulations (Klypin et al. 2011), cuspy subhalos are modeled with NFW profiles (Navarro et al. 1997):

$$
\rho(r)=\frac{\rho_{s}}{\left(\frac{r}{r_{s}}\right)\left(1+\frac{r}{r_{s}}\right)^{2}},
$$

where $\rho_{s}$ is the normalization and $r_{s}$ is the scale radius. For a given halo, $r_{s}$ and $\rho_{s}$ are assigned based on the $M_{200}$ at infall and the redshift-dependent mass-concentration relation from Prada et al. (2012) with 0.16 dex scatter (Diemer \& Kravtsov 2015). Cored halos are modeled with cored NFW profiles as:

$$
\rho(r)=\frac{\rho_{s}}{\left(1+\left(\frac{r}{r_{s}}\right)\right)^{3}} .
$$

For the cored halos, $\rho_{s}$ and $r_{s}$ are taken so that the $V_{\max }$ and $r_{\max }$ of the cored halo match their corresponding values for the corresponding cuspy halo. Although the physical processes behind core generation are under extensive debate, this model is generally consistent with observations, as well as SIDM models with low $\left(\sim 1 \mathrm{~cm}^{2} / \mathrm{g}\right)$ cross sections. Other models, motivated by the fact that supernova feedback can lower the dark matter density in the centers of halos (Di Cintio et al. 2014a; Madau et al. 2014; Pontzen \& Governato 2014; Read et al. 2016; Fitts et al. 2017; Zolotov et al. 2012), suggest smaller cores, closer to the galaxy half-light radius. We test the impact of different profile shapes on our analysis in Appendix A, including the Di Cintio et al. (2014a) profile; however as the mass profiles of halos with feedback-generated cores are not too different from our canonical halo, particularly for $r>r_{s}$ where most tidal radii occur, our conclusions are not very sensitive to the exact shape of cored halos at infall. Additionally, we note that the although Errani et al. (2018) tracks were modeled with a Dehnen (1993) profile (with an outer slope of 4 instead of 3), they should be applicable for NFW profiles given that the stellar component is well within the inner region of our halos for our systems. Thus, we remain agnostic to the mechanism producing cored halos, so long as the core is formed before the halo's infall onto the cluster.

Models including SIDM as well as baryonic effects fit Eqn. 6 well but evolve over time in the field, such that halos that fell into the cluster at earlier times have smaller cores (Kamada et al. 2017). While our procedure may overestimate the core sizes for the $54 \%$ of subhalos that fell in before $z=1$ (at which point core sizes are within $\sim 30 \%$ of their $z=0$ sizes; Kaplinghat et al. 2016), analysis in Appendix A demonstrates that our results are not particularly sensitive to the core size at infall, as long as the galaxy evolves along the cored tracks.

While this procedure is appropriate for the majority of lowmass subhalos (below $\sim 1 \times 10^{11} \mathrm{M}_{\odot}$ ), it may be inappropriate for higher mass subhalos. The simulations producing the Errani et al. (2018) tracks are exclusively set up in systems where the dark-matter density is higher than the stellar density at all radii.
This condition does not apply to all systems in our model, however, with many higher-mass systems exhibiting baryon-dominated centers. Although this is unlikely to affect the overall evolution of the dark-matter halo in response to tides (which is sensitive to the mass profile far away from the stellar component) it may affect the response of the stellar profile to tidal heating (which is sensitive to the potential felt by the stellar component). To model this possible effect, we treat systems with baryon-dominated centers as intermediate cases between cores and cusps, with $\gamma$ between 0 and 1. A conservative estimate for the appropriate value of $\gamma$ is determined using the ratio of stellar mass to dark-matter mass within the stellar half-light radius. If this quantity $\left(\delta_{1 / 2}\right)$ is less than $1.0, \gamma$ is set to 0 . If $\delta_{1 / 2}$ is greater than $2.0, \gamma$ is set to 1 . For values in between 1.0 and $2.0, \gamma$ is set to $\delta_{1 / 2}-1$. The $M_{*}$, final $/ M_{*}$, initial and $r_{e, \text { final }} / r_{e}$, initial tracks are interpolated to the appropriate value of $\gamma$ and the stellar profile is evolved according to the tracks for this intermediate $\gamma$. Given that the shape of the gravitational potential in the central regions of baryon-dominated systems in cored halos more resembles that of a cored halo than a cuspy one (as UDGs, and dwarf galaxies in general, have shallow stellar density profiles Faber \& Lin 1983; van Dokkum et al. 2015a; Yagi et al. 2016), this procedure provides a method for conservatively estimating the change in the stellar profile of baryon-dominated systems.

The combination of $M_{* \text {, final }} / M_{* \text {, initial }}$ and $r_{e}$, final $/ r_{e}$, initial determined from Equation 2 and the initial $M_{*}$ and $r_{e}$ values assigned in Section 2 establish the final $M_{*}$ and $r_{e}$ values that are observed at $z=0$. An example of the transition between the infall profile and the $z=0$ profile is illustrated in Figure 1. As the inner region of the cuspy halo is relatively unaffected by tidal stripping, the stellar profile is not significantly affected. On the other hand, stripping and heating are able to penetrate to the center of the cored halo, allowing for tidal heating in the central regions and an expansion of the stellar population.

In summary, our analysis proceeds as follows:

(i) Identify all subhalos around massive clusters in Illustris-dark at $z=0$.

(ii) Generate model cored and cuspy halo density profiles for each subhalo, based on $M_{\text {peak }}$ and the cosmological massconcentration relation.

(iii) Assign each halo a stellar mass (based on abundance-matching) and half-light radius (based on the dwarf-elliptical size-mass relation) before its infall onto the cluster.

(iv) Determine the degree of dark-matter stripping using the subhalo orbit and redshift-dependent host properties taken directly from the simulation. Concurrently change the subhalo density profiles using the Peñarrubia et al. (2010) tracks.

(v) Based on the amount of mass loss experienced by $z=0$, use the Errani et al. (2018) tracks to derive the final stellar mass and half-light radius.

\section{PROPERTIES OF TIDALLY-STRIPPED UDGS}

\subsection{Stellar Masses and Half-Light Radii}

Given the procedure outlined in Section 3, we determine the stellar masses and half-light radii of cluster satellites in the Illustris-dark simulation, following tracks for both cored and cuspy dark matter halos. Figure 2 illustrates the resulting satellite population of a $1.2 \times 10^{14} \mathrm{M}_{\odot}$ cluster alongside the UDGs observed in the Coma cluster $\left(M_{200}=2 \times 10^{15} \mathrm{M}_{\odot}\right)$ from Yagi et al. (2016) and dwarf- 

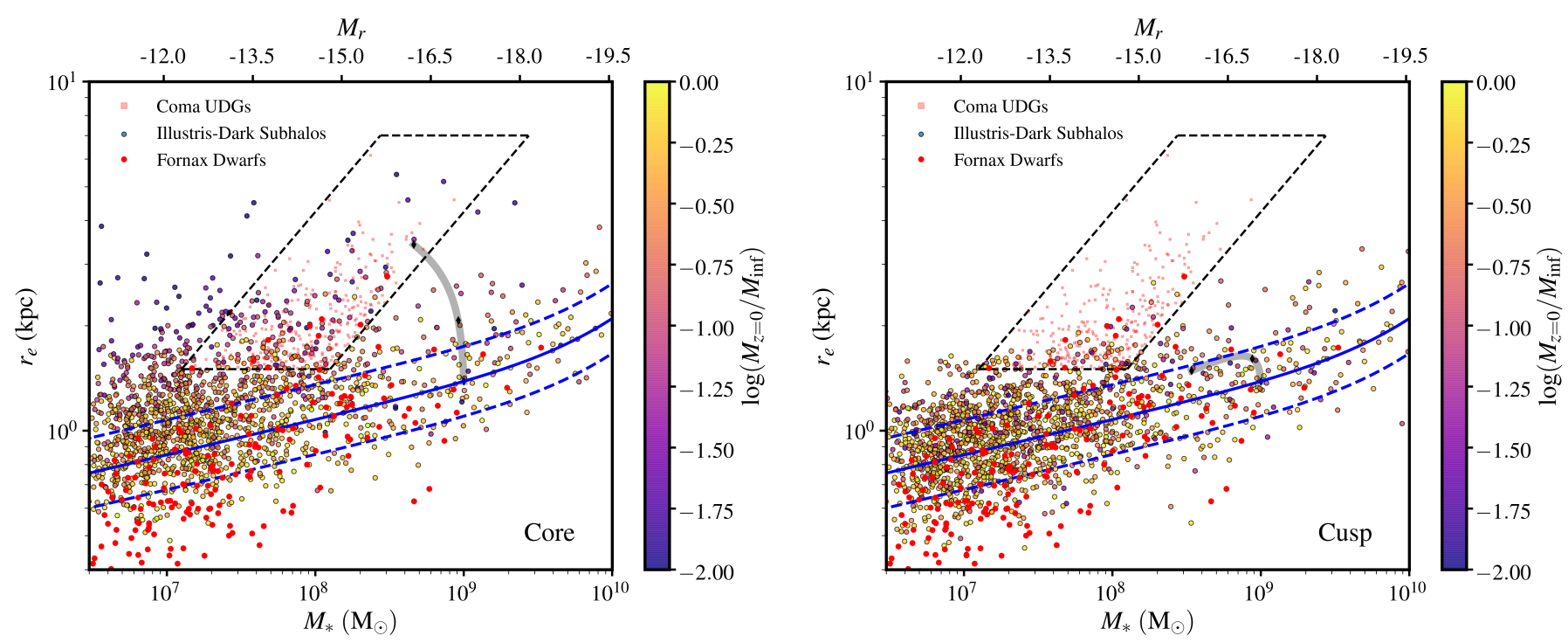

Figure 2. The relationship between stellar mass and half-light radius for both observed systems in the Coma cluster (red squares) and simulated objects (colored points) in cored (left) and cuspy (right) halos within a $M_{200}=2.4 \times 10^{14} \mathrm{M}_{\odot}$ cluster. The degree of mass loss experienced between infall and $z=0$ for cored and cuspy systems is illustrated by the coloring of each point. Blue solid and dashed lines indicate the starting mass-size relation and the associated $1 \sigma$ scatter that simulated systems are drawn from at infall, while the black dashed lines illustrate the UDG selection criteria. The thick grey line shows the evolution of a $10^{9} \mathrm{M}_{\odot}$ galaxy experiencing up to 99 percent mass loss, with black diamonds denoting the location of the galaxy at 0,90 , and 99 percent mass loss (evolving from low to high $r_{e}$, respectively). Also shown as red points are satellites in the Fornax cluster (Muñoz et al. 2015; Eigenthaler et al. 2018). Overall, cuspy halos do not experience the required mass loss to generate a substantial number of UDGs, whereas systems in cored subhalos produce UDGs with a range of sizes and stellar masses that broadly agree with observed UDG samples.

ellipticals from the Next Generation Fornax Survey ${ }^{1}$ (Muñoz et al. 2015; Eigenthaler et al. 2018). Galaxies in cored dark matter halos, which experience significantly more mass loss than those in cuspy halos, span a broad range of sizes, whereas galaxies in cuspy halos largely fall along the field size-mass relation.

In order to compare the properties of our simulated, tidallystripped UDGs with observational samples, we select UDGs as galaxies with $\Sigma_{*}=M_{*} /\left(\pi r_{e}^{2}\right)$ between $1.73 \times 10^{6}$ and $17.3 \times$ $10^{6} \mathrm{M}_{\odot} \mathrm{kpc}^{-2}$ and $r_{e}$ between 1.5 and $7 \mathrm{kpc}$ at $z=0$. The $r_{e}$ criterion is in line with existing UDG definitions (Koda et al. 2015; van der Burg et al. 2016), and the surface brightness criterion corresponds to $24\left\langle\langle\mu\rangle_{e}<26.5,{ }^{2}\right.$ where $\langle\mu\rangle_{e}$ is the average surface brightness within $r_{e}$ assuming an $r$-band mass-to-light ratio of 1.96 . This mass-to-light ratio, taken from Zibetti et al. (2009) assuming $g-r=0.689$, is subject to significant uncertainty due to a lack of deep near-IR photometry. ${ }^{3}$ However, cluster UDGs appear to have optical colors consistent with the red sequence (Yagi et al. 2016), so this assumption is not likely to significantly affect our results.

The stellar mass distribution of tidally-stripped UDGs, illustrated in Figure 3, peaks at $10^{8} \mathrm{M}_{\odot}$ for both cuspy and cored halos, consistent with observations (van der Burg et al. 2016). As shown in Figure 2, the UDG criteria at this mass is only $60 \%$ $(2.3 \sigma)$ away from the field size-mass relation, such that $\sim 1 \%$ of

1 The virial mass of Fornax is $M_{200}=7 \times 10^{13} \mathrm{M}_{\odot}$ (Schuberth et al. 2010).

2 Cosmological surface brightness dimming at the distance of Coma,

$\sim 0.1 \mathrm{mag} \operatorname{arcsec}^{-2}$, is taken into account when converting observed $\langle\mu\rangle_{e}$ to $\Sigma_{*}$.

3 We convert the CFHT colors to the SDSS filter set according to the following transformations:

$$
\begin{gathered}
g_{\text {CFHT }}=g_{\text {SDSS }}-0.153\left(g_{\text {SDSS }}-r_{\text {SDSS }}\right) \\
r_{\text {CFHT }}=r_{\text {SDSS }}-0.024\left(g_{\text {SDSS }}-r_{\text {SDSS }}\right) .
\end{gathered}
$$$$
\text { and }
$$

$10^{8} \mathrm{M}_{\odot}$ galaxies start off as UDGs simply due to scatter in the size-mass relation. Nevertheless, UDGs in cored halos distinguish themselves from those produced in cuspy halos, as they include a significant population of high-mass systems, in agreement with observations, whereas UDGs in cuspy halos do not. Because of the criteria used to define UDGs, these high-mass UDGs can only be created through significant tidal heating of more massive satellites (see Fig. 3.1) in cored halos.

On the other hand, the distributions of the half-light radii of UDGs produced in cored and cuspy halos differ more substantially. Figure 4 shows that the observed high- $r_{e}$ tail of the size distribution is reproduced in cored, but not in cuspy UDGs. While the size distribution of UDGs in cored halos follows $n \propto r^{-4.0}$, which is slightly steeper than the $n \propto r_{e}^{-3.4 \pm 0.19}$ found by van der Burg et al. (2016), the size distribution of UDGs in cuspy halos falls much more steeply than the observations (following $n \propto r_{e}^{-7.6}$ ). The fraction of cored UDGs with $r_{e}$ above $3 \mathrm{kpc}$ is $4.7 \%$, and the fraction of UDGs with $r_{e}$ above $5 \mathrm{kpc}$ is $0.4 \%$, compared with the observed fractions of $16.8 \%$ and $3.1 \%$ respectively. While our model does not quite produce the same population of extremely extended $(>4.5 \mathrm{kpc})$ systems found in observations, this population can easily be explained if we assume that they follow the size-mass relation for blue systems before stripping occurs.

However, under the unreasonable assumption that all systems follow the star-forming size-mass relation before stripping (see Sec. 2.1), the size distribution is significantly flatter than observed for both cored $\left(n \propto r^{-2.3}\right)$ and cuspy $\left(n \propto r^{-2.8}\right)$ halos and does not reproduce the observed dwarf-elliptical size distribution. Overall, the ability of tidal stripping of satellites in cored halos to reproduce the UDG size distribution from 1.5 to $>3 \mathrm{kpc}$ is strong evidence in favor of this formation mechanism. 


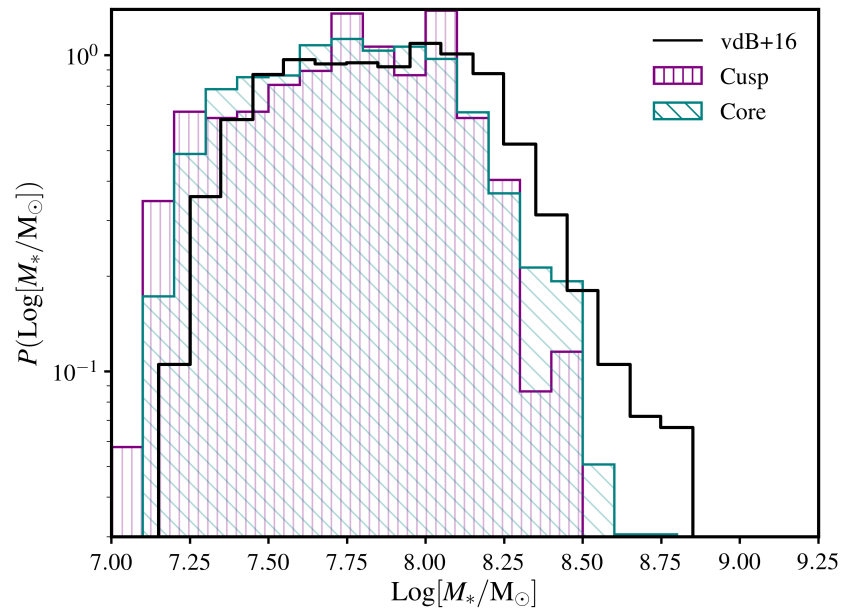

Figure 3. The relative mass distribution of tidally-stripped UDGs within cored (teal) and cuspy (purple) halos compared with the observed UDG mass function from van der Burg et al. (2016) (black). Both cuspy and cored distributions match the observed distribution remarkably well, peaking between $10^{7.5}$ and $10^{8} \mathrm{M}_{\odot}$. As shown in Figure 2, the sizes of galaxies within this mass range are only a few tenths of a dex away from the UDG criteria, such that little-to-no transformation is necessary to transform them into UDGs. Additionally, the large sizes of UDGs in cored profiles allow for a population of high-mass UDGs, similar to observations.

\subsection{Stellar Populations}

Given that tidally-stripped UDGs must have orbited the host cluster long enough to experience multiple pericentric passages, our analysis suggests that UDGs are primarily composed of old stellar populations. In particular, the largest UDGs, which in our formation scenario are formed after significant tidal stripping, should host significantly older stellar populations than smaller UDGs and nonUDG dwarf-ellipticals. As seen in Figure 2, the largest ( $r_{e} \gtrsim 3 \mathrm{kpc}$ ) UDGs have lost over 90 percent of their dark matter mass. On the other hand, smaller UDGs can be formed without significant mass loss, and may include younger stellar populations. If satellites are taken to be quenched $\sim 1$ Gyr after infall (Fillingham et al. 2015), our analysis predicts that the average stellar age of UDGs with $r_{e}$ between 1.5 and $3 \mathrm{kpc}$ is $4.8 \mathrm{Gyr}$ with the $10-90$ percentile spread $0.3-8.0 \mathrm{Gyr}$, but the age of UDGs with $r_{e}$ between 4.5 and $6 \mathrm{kpc}$ is $7.8 \mathrm{Gyr}$, with the $10-90$ percentile spread $6.2-9.1 \mathrm{Gyr}$. This prediction is consistent with the $\sim 10 \mathrm{Gyr}$ ages measured in large UDGs in Coma (Gu et al. 2017; Pandya et al. 2018; Ruiz-Lara et al. 2018; Ferre-Mateu et al. 2018), however, a more complete exploration of the size-age parameter space will provide better constraints on this model.

Recognizing that our simulated UDGs are born in low-mass halos $\left(\sim 10^{10}-10^{10.5} \mathrm{M}_{\odot}\right)$, we expect them to have low metallicities consistent with the classical low-mass dwarfs $([\mathrm{Fe} / \mathrm{H}] \sim-1.1$, Kirby et al. 2013). This aligns with spectral (Kadowaki et al. 2017; Gu et al. 2017; Ruiz-Lara et al. 2018; Ferre-Mateu et al. 2018) and color-based (Román \& Trujillo 2017b; Pandya et al. 2018) measurements of the stellar metallicities of UDGs.

\subsection{Dark Matter Halos}

Of particular interest when considering the formation of UDGs are the dark matter halos that host these galaxies. For example, the high

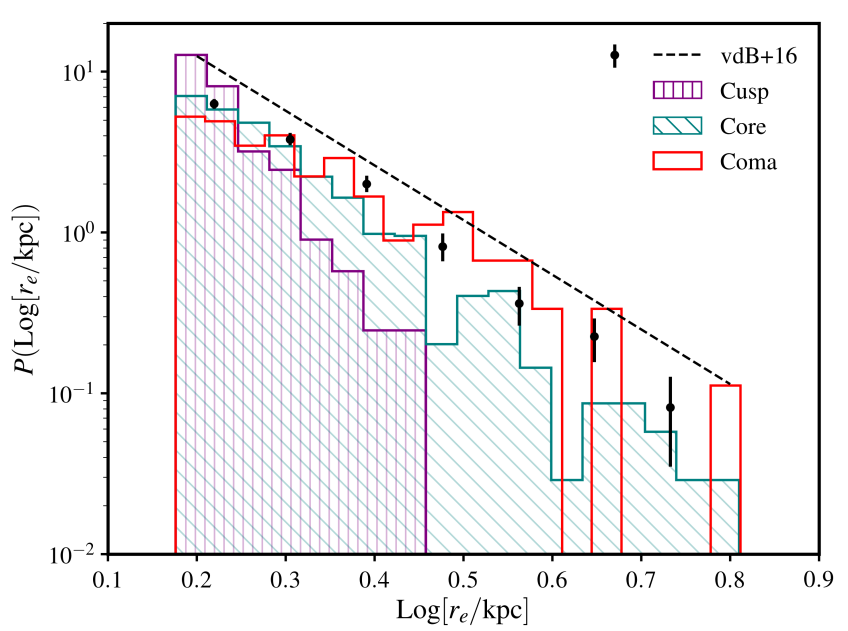

Figure 4. The distribution of $r_{e}$ for tidally-stripped UDGs within both cuspy (purple) and cored (teal) subhalos. Also shown, as black points, is the size distribution of UDGs within nearby clusters (and the fit to that distribution as the black line; van der Burg et al. (2016)), as well as the size distribution of Coma UDGs from Yagi et al. (2016) as the red histogram. The high- $r_{e}$ tail of the observed distribution is reproduced for UDGs in cored subhalos, but not for UDGs in cuspy subhalos. In particular, tidal stripping of galaxies in cuspy dark matter halos is not able to produce the population of large $\left(r_{e} \gtrsim\right.$ $3 \mathrm{kpc}$ ) UDGs. Although our model appears to underpredict the abundance of extremely extended ( $>4.5 \mathrm{kpc}$ ) systems, such systems would be expected in our model if they followed the size-mass relation for star-forming systems before stripping (see Sec. 2.1).

stellar velocity dispersion observed for DF 44 - implying a central dark matter fraction of $>98 \%$ - motivated the idea that UDGs are primarily formed in Milky Way-like halos that failed to form a substantial stellar mass (van Dokkum et al. 2015b). In our proposed scenario, however, UDGs are formed in similar dark matter halos as typical dwarf ellipticals, characterized by stellar velocity dispersions of $\sim 15 \mathrm{~km} \mathrm{~s}^{-1}$ within the half-light radius today. Although tidally-stripped UDGs experience significant dark matter mass loss, the mass loss is primarily in the halo outskirts (see Fig. 1), creating UDGs which contain similar amounts of dark matter within the half-light radius as typical dwarf galaxies. The average line-of-sight velocity dispersion of cored UDGs is $14 \mathrm{~km} \mathrm{~s}^{-1}$, with the $10-90$ percentile range extending from 9 to $23 \mathrm{~km} \mathrm{~s}^{-1}$.

For our analysis, we compute dispersions from the line-ofsight virial theorem (Merrifield \& Kent 1990) assuming a Plummer stellar profile. Nevertheless, given the uncharacteristic way in which UDGs fill their dark-matter halos, we have also carried out a systematic check of how well various dispersion-based mass estimators are able to determine the masses of UDGs, which have halflight radii similar to $r_{\max }$ and a stellar component which contributes significantly to the central density. This comparison was motivated by the analysis of Errani et al. (2018), who show that estimators of the form $M\left(a r_{e}\right)=b \sigma_{\mathrm{los}}^{2} r_{e} / G$ (e.g. Walker et al. 2009; Wolf et al. 2010) are systematically biased depending on the shape of halo density profile and the stellar segregation $\left(r_{e} / r_{\max }\right)$. In particular, we find that the above estimators underpredict the dispersions of UDGs (when compared with the line-of-sight Virial Theorem) by as much as $40 \%$ (i.e., masses inferred from UDG dispersions may be overestimated by a factor of 2). Regardless, we expect the dynamics of most UDGs to be more similar to field dwarfs than the Milky Way. 


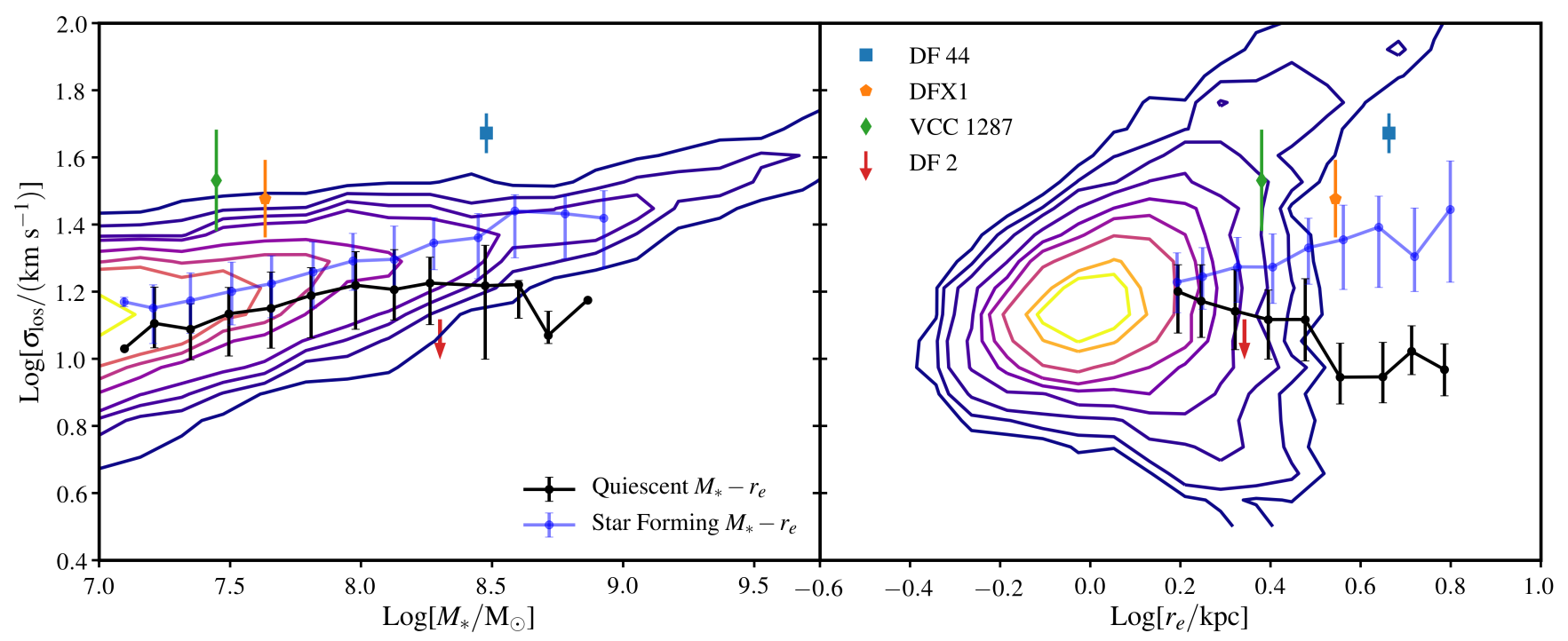

Figure 5. The relationship between line-of-sight velocity dispersion and final stellar mass (left) and final half-light radius (right), highlighting the field population as contours and the UDG population as points, with the $25-75 \%$ range illustrated with error bars. Black points illustrate the UDG population generated by our model assuming the size-mass relation for quiescent systems before stripping, whereas light blue points are generated assuming the starforming size-mass relation before stripping. Although UDGs are biased toward slightly lower velocity dispersions as a consequence of tidal stripping, they otherwise mimic the field relationships. Similarly, as UDGs which started on the star-forming size-mass relation have experienced less stripping than those starting on the quiescent size-mass relation, their dispersions are somewhat higher for high-mass UDGs. Also shown is the velocity dispersions of DF 44 (shown as the blue square), the globular cluster velocity dispersions VCC 1287 and DFX1 (shown as the orange pentagon and green diamond), and the upper limit on the globular cluster velocity dispersion of DF2 (as the red arrow). The existence of very dark-matter dominated objects like DF 44 and VCC 1287 may be explained if they lie slightly above the size-mass relation for star-forming systems (see Sec. 4.3).

A somewhat counterintuitive prediction of this analysis is that the mass within the half-light radius is not significantly altered by the stripping process for systems in cored halos. The galaxy expands as mass is lost in the center of the halo, keeping the mass within the half-light radius roughly constant (compared with the total amount of mass loss) and resulting in only a slight decrease in the predicted stellar velocity dispersion (Errani et al. 2015). Overall, UDGs in our analysis manifest a $\sim 0.07$ dex bias toward low $\sigma_{\text {los }}$ at a given $M_{*}$. Apart from this bias, the relationship between $\sigma_{\mathrm{los}}$ and $M_{*}$ for UDGs largely mirrors that of the field population, as dark-matter stripping occurs concurrently with stellar expansion (see Fig. 5). There is a notable lack of UDGs with high stellar mass and high velocity dispersion in our model, as the highest mass systems requires the most tidal stripping (see Fig. 2). This is in contrast with predictions from feedback models, in which UDGs with the highest stellar mass formed in the most massive halos at late times, resulting in higher velocity dispersions in the highest mass UDGs. Alternatively, if UDGs are formed in Milky Way-like halos, one would expect a uniformly high stellar velocity dispersion, regardless of $M_{*}$, again contrary to our predictions. We find that $\sigma_{\text {los }}$ decreases slightly at high $r_{e}$, in contrast with predictions from feedback models, which suggest a flat relationship between $r_{e}$ and $\sigma_{\text {los }}$. Despite the dramatic tidal effects experienced by these systems, we predict that the baryonic tracers of host dark-matter halos should not significantly deviate from those of field dwarfs.

This appears to be at odds with the large dispersion observed in DF 44 (van Dokkum et al. 2016). While none of the UDGs in our statistical analysis are this dark-matter dominated, we would not expect them to be present if they were significant outliers compared with the overall galaxy population at infall given the size of our simulated sample. We find that the size, stellar mass, and ve- locity dispersion of DF 44 can be explained if the infalling galaxy started out in a more concentrated halo and with a larger than usual half-light radius. As a specific example, consider a mock galaxy with a stellar mass of $7.3 \times 10^{8} \mathrm{M}_{\odot}$ and half-light radius of $3.7 \mathrm{kpc}$ that experiences $87 \%$ mass loss within $r_{\max }$ over the course of two pericentric passages. Should such a galaxy live in an $8.5 \times 10^{10} \mathrm{M}_{\odot}$ halo $(0.3 \sigma$ above what our abundance-matching prescription predicts) with a concentration $(c)$ of $18.3(\sim 2.7 \sigma$ above the cosmological mass-concentration relation) before it fell into Coma, the resulting halo would have a line-of-sight velocity dispersion of $42 \mathrm{~km} \mathrm{~s}^{-1}$ at $z=0$, consistent with the measured dispersion of DF $44 .{ }^{4}$ A similar scenario can explain the observed globular cluster dynamics of VCC 1287 and DFX1.

While the large sizes of these systems at infall lie well outside the predicted size-mass relation for quiescent systems $(3.7 \mathrm{kpc}$ is $>4 \sigma$ away from the quiescent size-mass relation), it is not far beyond the star-forming size-mass relation. In fact, this discrepancy $\left(r_{e}=3.7 \mathrm{kpc}\right.$ is $1.4 \sigma$ above the blue size mass relation for $M_{*}=3.5 \times 10^{8} \mathrm{M}_{\odot}$ ) may be lower if the intrinsic scatter in the size-mass relation is larger for dwarfs, as expected by some models as a consequence of star-formation feedback or dark-matter selfinteractions (Pontzen \& Governato 2014; Chan et al. 2018; Vogelsberger et al. 2014b) and in line with the observed presence of such large systems in the field (Leisman et al. 2017). While our use of the size-mass relation for red galaxies was chosen to jointly match the overall properties of the dwarf-elliptical and UDG populations in

\footnotetext{
${ }^{4}$ Placing DF 44 in a cored halo with an outer slope of 5 (see Sec. 3) and $M_{\mathrm{vir}}=10^{10} \mathrm{M}_{\odot}$ is consistent with the observed stellar dispersion. In contrast, van Dokkum et al. (2015b) place DF 44 in a $10^{12} \mathrm{M}_{\odot} \mathrm{NFW}$ halo to explain the stellar velocity dispersion.
} 
clusters (see Sec. 2.1), the presence of UDG progenitors that more closely follow the size-mass relation for star-forming systems before stripping may be necessary to produce the large dispersions of these UDGs. Overall, while a $M_{*}=3.5 \times 10^{8} \mathrm{M}_{\odot}$ and $r_{e}=3.7 \mathrm{kpc}$ system initially seems to be an unlikely outlier in our model, such large systems should not be unexpected, and have indeed been observed in the field (Leisman et al. 2017).

Similarly, as tidal stripping preferentially removes mass in the halo outskirts, our scenario allows for the possibility of UDGs that appear baryon-dominated in their outskirts, like the recently identified UDG DF2 van Dokkum et al. (2018). In our scenario, if a dwarf with $M_{*}=2 \times 10^{8} \mathrm{M}_{\odot}$ and $r_{e}=0.3 \mathrm{kpc}$ in a cored $M_{\text {vir }}=5 \times 10^{10} \mathrm{M}_{\odot}, c=6.7$ halo lost $99.97 \%$ of its mass, it would have $M_{*}=1.8 \times 10^{8} \mathrm{M}_{\odot}$, and $r_{e}=2.2 \mathrm{kpc}$ at $z=0$. The resulting dark-matter halo, would have a mass of only $9.1 \times 10^{7} \mathrm{M}_{\odot}$ and a low velocity dispersion consistent with the observations (Martin et al. 2018).

In summary, while systems like DF 2 and DF 44 should be rare among the UDG population in our scenario, the observed properties of these objects are consistent with unusual galaxies made more extreme through tidal stripping.

\subsection{Tidal Features}

A unique prediction of this formation scenario is that UDGs will be elongated along their orbital axes and exhibit distinct tidal features. Simulations of tidally-stripped systems find that axis ratios of galaxies in cored halos increase significantly after multiple pericentric passages (Errani et al. 2015). Furthermore, S-shaped tidal features have been observed in satellites with recent tidal interactions (Choi et al. 2002; Odenkirchen et al. 2003) as well as simulations of close host-satellite interactions (Johnston et al. 2002, 2008). This prediction is supported by the fact that UDGs in Coma tend to be aligned towards the cluster center (Yagi et al. 2016; Burkert 2017). However, although tidal features have been observed around some UDGs (Toloba et al. 2016; Müller et al. 2018), Mowla et al. (2017) find an absence of S-shaped tidal features out to $7 \mathrm{kpc}$ around UDGs in Coma. This absence of S-shaped tidal features establishes a lack of recent stellar stripping among most Coma UDGs. However, such features are not expected to persist long after a pericentric passage. Specifically, Peñarrubia et al. (2009) find that such features expand away from the satellite at roughly half the rate of the stellar velocity dispersion. Assuming that S-shaped tidal features expand away from UDGs at this rate, they would reach $7 \mathrm{kpc}$ from the UDG center $0.68 \mathrm{Gyr}$ after a pericentric passage. Among tidally-stripped UDG in Illustris-dark, $13 \%$ experienced a pericentric passage in the past $0.68 \mathrm{Gyr}$, suggesting that S-shaped tidal features should be observable in this fraction of UDGs in Coma, marginally consistent with the observations of Mowla et al. (2017). Furthermore, the S-shaped features searched for by Mowla et al. (2017) are expected to become aligned along the orbital direction quickly (Peñarrubia et al. 2009), significantly reducing the predicted distortion of UDG morphologies.

\subsection{Radial Distribution}

The spatial distribution of UDGs within clusters can provide a valuable piece of information regarding UDG formation and evolution. Among Illustris subhalos, those hosting tidally-stripped UDGs largely mirror the subhalo distribution, with a slight $(\sim 4 \%)$ in the cluster centers $\left(0.1 \times R_{200}\right)$. Given that $53 \%$ of tidally-stripped

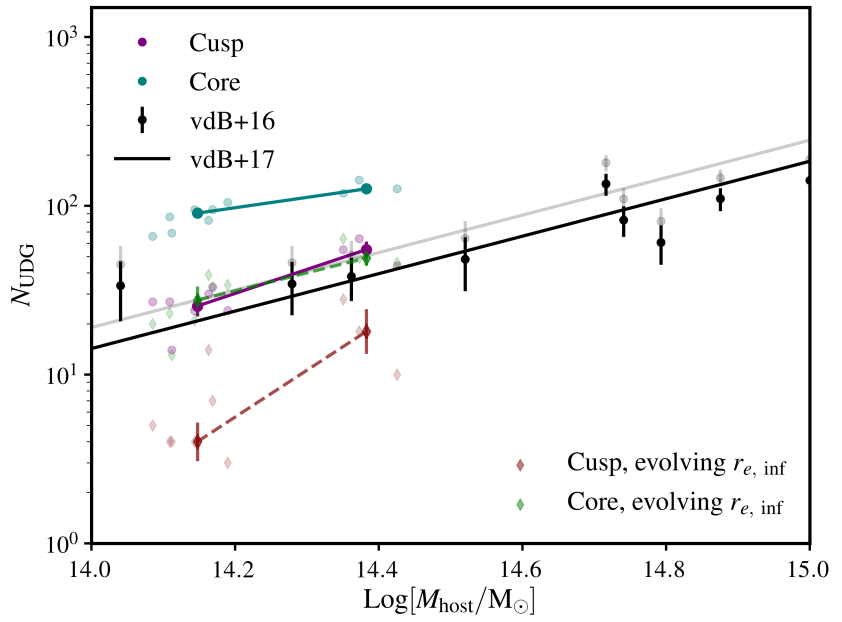

Figure 6. The abundance of tidally-stripped UDGs as a function of host halo mass for UDGs hosted in cuspy (teal) and cored (purple) dark-matter halos. For comparison, the black points and back line correspond to observed UDG abundances from van der Burg et al. (2016) and the fit to the UDG abundance as a function of halo mass from van der Burg et al. (2017), respectively. For our fiducial model, the abundance of cored UDGs is a factor of 4 greater than the observed UDG abundance and the abundance of cuspy UDGs matches the observed UDG abundance. However, if the starting sizes evolve with redshift according to $(1+z)^{-0.6}$, the abundance of cored UDGs (as shown by the light teal diamonds) agrees with the observed abundance within and the abundance of cuspy UDGs (as shown by the purple diamonds) significantly underestimates the UDG abundance.

UDGs in cored halos experience two or fewer pericentric passages, it makes sense that tidally-stripped UDGs are not exclusively found in cluster centers.

On the other hand, our finding that UDGs tend to be slightly overabundant in cluster centers relative to subhalos of similar mass is inconsistent with the observation of a deficit of UDGs in cluster centers (van Dokkum et al. 2015a; van der Burg et al. 2016). This may point to a breakdown of our model in the most extremely disrupted systems. Our analysis produces a number of remarkably tidally-stripped ( $>99 \%$ mass loss) systems in the central regions of the clusters. Our model for tidal stripping is not as well constrained for systems with this degree of mass loss, such that UDGs with a such a high degree of stripping may loose mass more quickly than our model predicts. For example, the assumption that tidal stripping only occurs at pericenter may break down in the central regions as tidal effects become more important throughout the orbit. In this case, we may underestimating how many UDGs in cluster centers are stripped to the point that they are below our detection threshold or completely destroyed.

\subsection{Abundance}

The increase in UDG abundance with increasing cluster mass (van der Burg et al. 2016, 2017) provides a powerful constraint on the strength of environmental processes in shaping the UDG population. For instance, van der Burg et al. (2017) find that UDG abundance increases with increasing host mass as $N=19 \times$ $\left(M_{200} / 10^{14} \mathrm{M}_{\odot}\right)^{1.11}$, motivating an environmental-dependent formation mechanism. In van der Burg et al. (2017), the UDG abundance is determined by subtracting the abundance of UDGs surrounding clusters by the UDG abundance measured using the same 
procedure on a random region of the sky. However, this subtraction still overestimates the abundance of cluster satellites by counting objects infalling onto the cluster. To estimate the magnitude of this effect, we measure the ratio between the number of halos that would be counted as cluster members in the van der Burg et al. (2017) sample and the true satellite abundance in the Illustris-dark simulation. In van der Burg et al. (2017), satellites are identified as galaxies within the projected $R_{200}$ of the cluster and with a lineof-sight velocity within $5 \sigma$ of the cluster, where $\sigma$ is the velocity dispersion of the cluster from Sifón et al. (2015). We find that the van der Burg et al. (2017) abundance measurements overestimate the true abundance by a factor of 1.3 , with no significant dependence on either subhalo mass or cluster mass. Thus, we take the measured UDG abundance divided by this factor of 1.3 as the true UDG abundance within $R_{200}$.

Figure 6 shows the relationship between the abundance of tidally-stripped UDGs and host mass found within our analysis. As expected, the abundance of tidally-stripped UDGs increases with increasing halo mass; we find $N=67 \times\left(M_{200} / 10^{14} \mathrm{M}_{\odot}\right)^{0.76}$ for cored halos and $N=18 \times\left(M_{200} / 10^{14} \mathrm{M}_{\odot}\right)^{1.24}$ for cuspy halos. This model overproduces the UDG abundance by a factor of 3.8 for cored halos and $41 \%$ for cuspy halos. However, if the starting sizemass relation evolves weakly with redshift, following $(1+z)^{-0.6}$, the predicted abundance matches the observed for cored halos and the UDG size distribution is relatively unchanged (see Appendix B). Furthermore, the final size-mass relation for cored subhalos in this case is actually more consistent with the observed size distribution of dwarfs $\left(5 \times 10^{7}<M_{*} / M_{\odot}<2 \times 10^{8}\right)$ in the Fornax Cluster (Muñoz et al. 2015) than with the fiducial size-mass relation (KS-test $p$ value of 0.4 compared with 0.002 ).

Among clusters in our sample, the UDG abundance is a relatively constant fraction of the total subhalo abundance for both cored and cuspy subhalos, in contrast with the observed superlinear increase in UDG abundance with halo mass. However, the comparatively narrow range in host halo mass probed by this analysis limits our ability to compare this trend with the observed relation. If we make the assumption that the UDG fraction depends on the host mass within $200 \mathrm{kpc}$ (the mean pericenter distance for among our sample), it is possible to extrapolate the $N_{\mathrm{UDG}}-M_{\text {host }}$ relation. Within our sample, we find:

$$
\log \left\langle\frac{N_{\mathrm{UDG}}}{N_{\mathrm{sub}>10^{9.8} \mathrm{M}_{\odot}}}\right\rangle=0.76 \log \left[\frac{M_{\mathrm{host}}(200 \mathrm{kpc})}{10^{13} \mathrm{M}_{\odot}}\right]-1.39
$$

where $N_{\mathrm{UDG}} / N_{\text {sub }>10^{9.8}} \mathrm{M}_{\odot}$ is the fraction of subhalos with infall masses $>10^{9.8} \mathrm{M}_{\odot}$ that become UDGs and $M_{\text {host }}(200 \mathrm{kpc})$ is the host mass within $200 \mathrm{kpc}$. Extrapolating this relation down the hosts of $10^{12} \mathrm{M}_{\odot}$, assuming the subhalo mass function of Jiang \& van den Bosch (2016) and the Prada et al. (2012) massconcentration relation, this result suggests $N_{\mathrm{UDG}} \propto M_{\text {host }}^{1.2}$, roughly consistent with the observed trend in this mass range. Ultimately, although our fiducial model does not match the observed UDG abundance exactly, it is roughly consistent with observations.

Notably, as this method of UDG formation requires subhalos to orbit a massive cluster for many Gyr, it suggests that the relative UDG abundance is lower among recently-formed clusters. This suggests that more relaxed clusters and clusters with higher concentration values should have a higher UDG abundance than less relaxed clusters and clusters with lower concentration values of similar mass. Additionally, this suggests that the relative abundance of UDGs decreases with increasing $z$ - we find that the UDG abundance has decreased by $83 \%$ by $z=0.5$ (whereas the cluster subhalo abundance has decreased by only $24 \%$ ).

\section{CONCLUSIONS}

In this work, we have shown that tidal stripping of dwarf galaxies within clusters is able to reproduce the observed properties and abundance of UDGs remarkably well. The principal results of our analysis are as follows:

- In our modeling of tidal stripping, we find that cuspy halo do not experience the required mass loss to generate a substantial number of large cluster UDGs. In contrast, tidal forces acting upon dwarf galaxies hosted by $\sim 10^{10}-10^{11} \mathrm{M}_{\odot}$ cored halos do produce a cluster UDG population with properties (e.g. stellar mass, size, metallicity, etc.) that broadly agree with observed UDG samples.

- The distribution of observed half-light radii for local UDGs is reproduced by that of tidally-stripped UDGs in cored dark matter halos. In particular, the most extended UDGs with $r_{e}>3 \mathrm{kpc}$, which observations estimate to be $\sim 11-17 \%$ of the cluster UDG population, comprise $5 \%$ of our tidally-stripped, simulated sample.

- The abundance of tidally-stripped UDGs in cored halos increases with increasing cluster mass according to $N \propto M_{\text {host }}^{0.76}$, roughly consistent with the observed abundance.

- A number of concrete predictions arise from our model for UDG formation:

- While small UDGs have a range of stellar ages, the largest tidally-stripped UDGs $\left(r_{e}>3 \mathrm{kpc}\right)$ should host significantly older stellar populations, with $89 \%$ of such systems $>4 \mathrm{Gyr}$ old.

- Dark matter halos hosting UDGs have similar masses to typical dwarfs of the same mass. They should be more centrally concentrated than dwarfs at the same mass because dark matter is preferentially lost in the halo outskirts.

- At fixed host mass, UDG abundance should be correlated with cluster age/concentration and inversely correlated with redshift.

The main uncertainty of this model is the initial sizedistribution of dwarf galaxies before they experience tidal stripping and heating - the difference between the star-forming and quiescent size-mass relation can be as much 0.2 dex within our mass range. Compounding this difference with any possible redshift evolution and the different amounts of intrinsic scatter, this uncertainty easily has the largest effect on our analysis, and we advocate for further observational and theoretical work into investigating the processes processes involved in transforming large dwarf-irregular galaxies to smaller dwarf-ellipticals. Beyond that, the treatment of tidal stripping and heating among systems with baryon-dominated centers as well as systems with extreme mass loss and needs significant further investigation. In particular, the population of extremely large UDGs (i.e. those underproduced by our model) is composed of systems with $>99 \%$ mass loss - beyond the bounds of the Errani et al. (2018) tracks. 
We have presented a scenario for the formation of ultra-diffuse galaxies in clusters motivated by the presence of cores in field dwarf galaxies. We have shown that this scenario provides a compelling explanation for the abundance and radial alignment of ultradiffuse galaxies and it makes concrete predictions that can be tested in the near future.

\section{ACKNOWLEDGMENTS}

The authors are grateful to Remco van der Burg for helpful discussions related to the observed abundance of UDGs. Additionally, TMC would like to thank Andrew Graus for many helpful discussions regarding $N$-body simulations and Sheldon Campbell for his help with the analysis. Lastly, we are grateful to the anonymous reviewer, whose suggestions greatly improved this paper. This work was supported in part by NSF grants AST-1518257 and PHY1620638. Support for this work was provided by NASA through grants (AR-13242 and AR-14289) from the Space Telescope Science Institute, which is operated by the Association of Universities for Research in Astronomy, Inc., under NASA contract NAS 5-26555.

This research made use of Astropy, a community-developed core Python package for Astronomy (Astropy Collaboration et al. 2013). Additionally, the Python packages NumPy (Walt et al. 2011), iPython (Prez \& Granger 2007), SciPy (Jones et al. 2001), and matplotlib (Hunter 2007) were utilized for the majority of our data analysis and presentation.

\section{REFERENCES}

Agertz O., Kravtsov A. V., 2016, ApJ, 824, 79

Amorisco N. C., 2018, MNRAS, 475, L116

Amorisco N. C., Loeb A., 2016, MNRAS, 459, L51

Amorisco N. C., Monachesi A., White S. D. M., 2016, preprint, (arXiv: 1610.01595)

Amorisco N. C., Monachesi A., Agnello A., White S. D. M., 2018, MNRAS, 475,4235

Astropy Collaboration et al., 2013, A\&A, 558, A33

Beasley M. A., Trujillo I., 2016, ApJ, 830, 23

Beasley M. A., Romanowsky A. J., Pota V., Navarro I. M., Martinez Delgado D., Neyer F., Deich A. L., 2016, ApJ, 819, L20

Behroozi P. S., Wechsler R. H., Wu H.-Y., 2013a, ApJ, 762, 109

Behroozi P. S., Wechsler R. H., Conroy C., 2013b, ApJ, 770, 57

Bennet P., Sand D. J., Zaritsky D., Crnojević D., Spekkens K., Karunakaran A., 2018, ApJ, 866, L11

Bryan G. L., Norman M. L., 1998, ApJ, 495, 80

Burkert A., 2017, ApJ, 838, 93

Chan T. K., Kereš D., Wetzel A., Hopkins P. F., Faucher-Giguère C.-A., ElBadry K., Garrison-Kimmel S., Boylan-Kolchin M., 2018, MNRAS,

Choi P. I., Guhathakurta P., Johnston K. V., 2002, AJ, 124, 310

Conselice C. J., 2018, Research Notes of the American Astronomical Society, 2, 43

Creasey P., Sameie O., Sales L. V., Yu H.-B., Vogelsberger M., Zavala J., 2017, MNRAS, 468, 2283

Dehnen W., 1993, MNRAS, 265, 250

Di Cintio A., Brook C. B., Macciò A. V., Stinson G. S., Knebe A., Dutton A. A., Wadsley J., 2014a, MNRAS, 437, 415

Di Cintio A., Brook C. B., Dutton A. A., Macciò A. V., Stinson G. S., Knebe A., 2014b, MNRAS, 441, 2986

Di Cintio A., Brook C. B., Dutton A. A., Macciò A. V., Obreja A., Dekel A., 2017, MNRAS, 466, L1

Diemer B., Kravtsov A. V., 2015, ApJ, 799, 108

Driver S. P., et al., 2011, MNRAS, 413, 971
Eigenthaler P., et al., 2018, ApJ, 855, 142

El-Badry K., Wetzel A., Geha M., Hopkins P. F., Kereš D., Chan T. K., Faucher-Giguère C.-A., 2016, ApJ, 820, 131

Elbert O. D., Bullock J. S., Kaplinghat M., Garrison-Kimmel S., Graus A. S., Rocha M., 2018, ApJ, 853, 109

Errani R., Peñarrubia J., Tormen G., 2015, MNRAS, 449, L46

Errani R., Peñarrubia J., Laporte C. F. P., Gómez F. A., 2017, MNRAS, 465, L59

Errani R., Peñarrubia J., Walker M. G., 2018, preprint, (arXiv: 1805.00484)

Faber S. M., Lin D. N. C., 1983, ApJ, 266, L17

Fattahi A., Navarro J. F., Frenk C. S., Oman K. A., Sawala T., Schaller M., 2018, MNRAS, 476, 3816

Ferre-Mateu A., et al., 2018, preprint, (arXiv: 1801.09695)

Fillingham S. P., Cooper M. C., Wheeler C., Garrison-Kimmel S., BoylanKolchin M., Bullock J. S., 2015, MNRAS, 454, 2039

Fillingham S. P., Cooper M. C., Pace A. B., Boylan-Kolchin M., Bullock J. S., Garrison-Kimmel S., Wheeler C., 2016, MNRAS, 463, 1916

Fitts A., et al., 2017, MNRAS, 471, 3547

Geha M., Blanton M. R., Yan R., Tinker J. L., 2012, ApJ, 757, 85

Grogin N. A., et al., 2011, ApJS, 197, 35

Gu M., et al., 2017, preprint, (arXiv:1709.07003)

Hunter J. D., 2007, Computing in Science \& Engineering, 9, 90

Janssens S., Abraham R., Brodie J., Forbes D., Romanowsky A. J., van Dokkum P., 2017, ApJ, 839, L17

Jiang F., van den Bosch F. C., 2016, MNRAS, 458, 2848

Johnston K. V., Choi P. I., Guhathakurta P., 2002, AJ, 124, 127

Johnston K. V., Bullock J. S., Sharma S., Font A., Robertson B. E., Leitner S. N., 2008, ApJ, 689, 936

Jones E., Oliphan T., Peterson P., et al., 2001, SciPy: Open source scientific tools for Python, http://www.scipy.org/

Jones M. G., Papastergis E., Pandya V., Leisman L., Romanowsky A. J., Yung L. Y. A., Somerville R. S., Adams E. A. K., 2017, preprint, (arXiv: 1712.01855)

Kadowaki J., Zaritsky D., Donnerstein R. L., 2017, ApJ, 838, L21

Kamada A., Kaplinghat M., Pace A. B., Yu H.-B., 2017, Physical Review Letters, 119, 111102

Kaplinghat M., Tulin S., Yu H.-B., 2016, Physical Review Letters, 116, 041302

Kazantzidis S., Mayer L., Callegari S., Dotti M., Moustakas L. A., 2017, ApJ, 836, L13

Kirby E. N., Cohen J. G., Guhathakurta P., Cheng L., Bullock J. S., Gallazzi A., 2013, ApJ, 779, 102

Klypin A. A., Trujillo-Gomez S., Primack J., 2011, ApJ, 740, 102

Koda J., Yagi M., Yamanoi H., Komiyama Y., 2015, ApJ, 807, L2

Koekemoer A. M., et al., 2011, ApJS, 197, 36

Kravtsov A. V., Vikhlinin A. A., Meshcheryakov A. V., 2018, Astronomy Letters, 44,8

Kuzio de Naray R., McGaugh S. S., de Blok W. J. G., 2008, ApJ, 676, 920

Lange R., et al., 2015, MNRAS, 447, 2603

Laporte C. F. P., Agnello A., Navarro J. F., 2018, preprint, (arXiv: 1804.04139)

Lee M. G., Kang J., Lee J. H., Jang I. S., 2017, ApJ, 844, 157

Leisman L., et al., 2017, ApJ, 842, 133

Liske J., et al., 2015, MNRAS, 452, 2087

Madau P., Shen S., Governato F., 2014, ApJ, 789, L17

Martin N. F., et al., 2016, ApJ, 833, 167

Martin N. F., Collins M. L. M., Longeard N., Tollerud E., 2018, preprint, (arXiv: 1804.04136)

Merrifield M. R., Kent S. M., 1990, in Bulletin of the American Astronomical Society. p. 744

Merritt A., van Dokkum P., Danieli S., Abraham R., Zhang J., Karachentsev I. D., Makarova L. N., 2016, ApJ, 833, 168

Mihos J. C., et al., 2015, ApJ, 809, L21

Mowla L., van Dokkum P., Merritt A., Abraham R., Yagi M., Koda J., 2017, ApJ, 851, 27

Muñoz R. P., et al., 2015, ApJ, 813, L15

Müller O., Jerjen H., Binggeli B., 2018, preprint, (arXiv: 1802.08657) 
Navarro J. F., Frenk C. S., White S. D. M., 1997, ApJ, 490, 493

Nelson D., et al., 2015, Astronomy and Computing, 13, 12

Odenkirchen M., et al., 2003, AJ, 126, 2385

Ogiya G., 2018, preprint, (arXiv: 1804.06421)

Oh S.-H., de Blok W. J. G., Brinks E., Walter F., Kennicutt Jr. R. C., 2011, AJ, 141, 193

Oh S.-H., et al., 2015, AJ, 149, 180

Oman K. A., et al., 2015, MNRAS, 452, 3650

Ordenes-Briceño Y., et al., 2018, preprint, (arXiv: 1803.10784)

Pandya V., et al., 2018, ApJ, 858, 29

Peñarrubia J., Navarro J. F., McConnachie A. W., 2008, ApJ, 673, 226

Peñarrubia J., Navarro J. F., McConnachie A. W., Martin N. F., 2009, ApJ, 698,222

Peñarrubia J., Benson A. J., Walker M. G., Gilmore G., McConnachie A. W., Mayer L., 2010, MNRAS, 406, 1290

Peng E. W., Lim S., 2016, ApJ, 822, L31

Plummer H. C., 1911, MNRAS, 71, 460

Pontzen A., Governato F., 2014, Nature, 506, 171

Prada F., Klypin A. A., Cuesta A. J., Betancort-Rijo J. E., Primack J., 2012, MNRAS, 423, 3018

Prez F., Granger B. E., 2007, Computing in Science \& Engineering, 9, 21

Read J. I., Agertz O., Collins M. L. M., 2016, MNRAS, 459, 2573

Rodriguez-Gomez V., et al., 2015, MNRAS, 449, 49

Roediger J. C., et al., 2017, ApJ, 836, 120

Román J., Trujillo I., 2017a, MNRAS, 468, 703

Román J., Trujillo I., 2017b, MNRAS, 468, 4039

Ruiz-Lara T., et al., 2018, MNRAS,

Safarzadeh M., Scannapieco E., 2017, ApJ, 850, 99

Schuberth Y., Richtler T., Hilker M., Dirsch B., Bassino L. P., Romanowsky A. J., Infante L., 2010, A\&A, 513, A52

Shi D. D., et al., 2017, ApJ, 846, 26

Sifón C., Hoekstra H., Cacciato M., Viola M., Köhlinger F., van der Burg R. F. J., Sand D. J., Graham M. L., 2015, A\&A, 575, A48

Sifón C., van der Burg R. F. J., Hoekstra H., Muzzin A., Herbonnet R., 2018, MNRAS, 473, 3747

Smith Castelli A. V., Faifer F. R., Escudero C. G., 2016, A\&A, 596, A23

Spekkens K., Karunakaran A., 2018, ApJ, 855, 28

Springel V., White S. D. M., Tormen G., Kauffmann G., 2001, MNRAS, 328,726

Springel V., et al., 2008, MNRAS, 391, 1685

Toloba E., et al., 2016, ApJ, 816, L5

Toloba E., et al., 2018, ApJ, 856, L31

Trujillo I., Román J., Filho M., Sánchez Almeida J., 2017, ApJ, 836, 191

Venhola A., et al., 2017, A\&A, 608, A142

Vogelsberger M., et al., 2014a, MNRAS, 444, 1518

Vogelsberger M., Zavala J., Simpson C., Jenkins A., 2014b, MNRAS, 444, 3684

Walker M. G., Mateo M., Olszewski E. W., Peñarrubia J., Wyn Evans N., Gilmore G., 2009, ApJ, 704, 1274

Walt S. v. d., Colbert S. C., Varoquaux G., 2011, Computing in Science \& Engineering, 13, 22

Wheeler C., Phillips J. I., Cooper M. C., Boylan-Kolchin M., Bullock J. S., 2014, MNRAS, 442, 1396

Wheeler C., et al., 2017, MNRAS, 465, 2420

Williams R. P., et al., 2016, MNRAS, 463, 2746

Wittmann C., et al., 2017, MNRAS, 470, 1512

Wolf J., Martinez G. D., Bullock J. S., Kaplinghat M., Geha M., Muñoz R. R., Simon J. D., Avedo F. F., 2010, MNRAS, 406, 1220

Yagi M., Koda J., Komiyama Y., Yamanoi H., 2016, ApJS, 225, 11

Zibetti S., Charlot S., Rix H.-W., 2009, MNRAS, 400, 1181

Zolotov A., et al., 2012, ApJ, 761, 71

van Dokkum P. G., Abraham R., Merritt A., Zhang J., Geha M., Conroy C., 2015a, ApJ, 798, L45

van Dokkum P. G., et al., 2015b, ApJ, 804, L26

van Dokkum P., et al., 2016, ApJ, 828, L6

van Dokkum P., et al., 2017, ApJ, 844, L11

van Dokkum P., et al., 2018, Nature, 555, 629

van den Bosch F. C., 2017, MNRAS, 468, 885

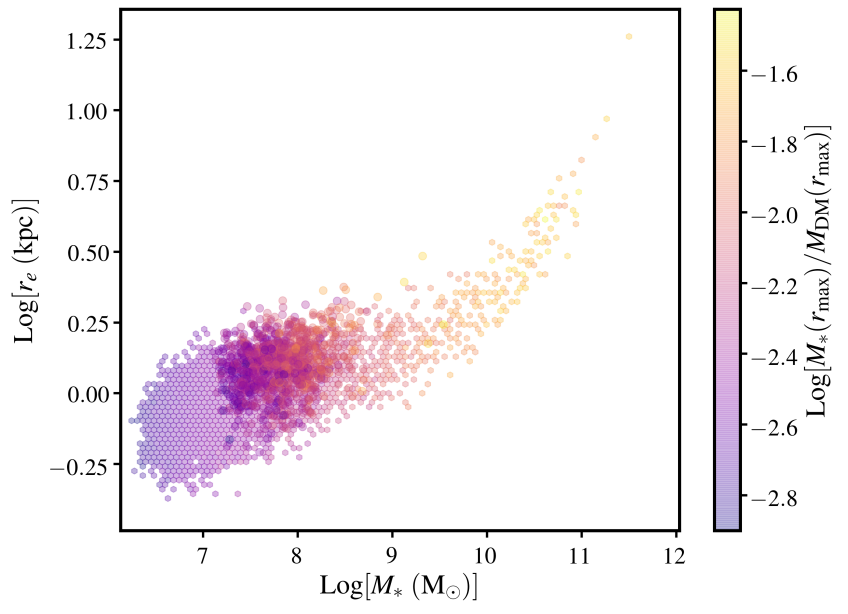

Figure A1. The size-stellar mass relation at infall for our simulated systems in Illustris-dark, color-coded by the stellar to dark matter mass ratio within $r_{\text {max }}$, with systems that evolve into UDGs highlighted. For all systems, the baryonic mass represents a very small fraction of the dark-matter mass.

van den Bosch F. C., Jiang F., Campbell D., Behroozi P., 2016, MNRAS, 455,158

van den Bosch F. C., Ogiya G., Hahn O., Burkert A., 2018, MNRAS, 474, 3043

van der Burg R. F. J., Muzzin A., Hoekstra H., 2016, A\&A, 590, A20

van der Burg R. F. J., et al., 2017, A\&A, 607, A79

van der Wel A., et al., 2014, ApJ, 788, 28

\section{APPENDIX A: CONSIDERING THE EFFECTS OF BARYONS ON CORED DARK-MATTER HALOS}

In this Appendix, we discuss in more detail how our analysis is affected by the presence of baryons in cored halos, particularly when considering baryon-dominated systems (see Sec. 3).

Our assumption that baryon-dominated systems undergo the same dark-matter stripping as dark matter-dominated systems is motivated by the fact that the inclusion of baryonic mass has a negligible impact on the $V_{\max }$ and $r_{\max }$ of a halo (see Fig. A1), although they could dominate within the half-light radius for some progenitors. Nevertheless, the presence of baryons may cause the halo to be more concentrated. Observations are consistent with a range of core sizes spanning from the NFW scale radius to the galaxy's half-light radius (Oman et al. 2015; Oh et al. 2015), and this wide variation has been observed in models including SIDM and baryons (Vogelsberger et al. 2014b; Kamada et al. 2017; Creasey et al. 2017) as well as baryonic feedback (Fitts et al. 2017; Di Cintio et al. 2014a). Our cored model, taken to match models including SIDM and baryons for galaxies in our sample (Kaplinghat et al. 2016; Kamada et al. 2017; Elbert et al. 2018), produces cores that extend to the scale radius of the halo. Nevertheless, we test how varying the core size affects our results by rerunning our analysis with the halos at infall generated by following the prescription of Di Cintio et al. (2014b), which is designed to fit halos affected by baryonic feedback with a mass-dependent profile shape. For the $r_{e}$ and $M_{*}$ tracks, we take $\gamma$ to be the larger of $\gamma$ determined from $\delta_{1 / 2}$ or $\gamma$ from Di Cintio et al. (2014b). As these halos have a shallower outer slope than our fiducial model, they experience slightly more mass loss overall. However, as they are more cuspy than our fiducial model, they 


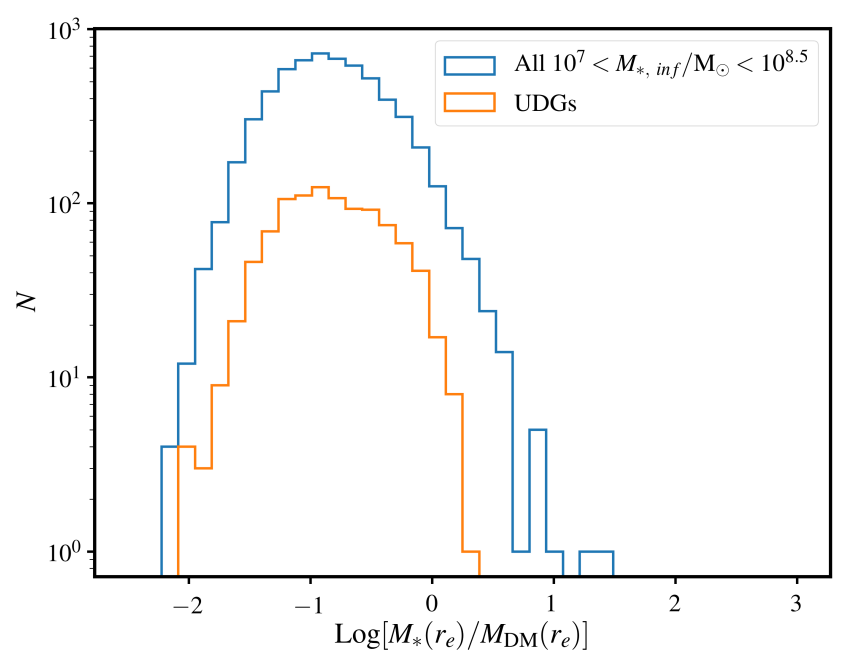

Figure A2. The distribution of $\delta_{1 / 2}$ for all simulated systems in Illustrisdark within the mass range $\left(10^{7}<M_{*} / M_{\odot}<10^{8.5}\right.$, blue line $)$ and for just those systems the become UDGs (orange line). Overall, baryon-dominated systems are a negligible component of the starting population, and the exact treatment of them does not significantly change the conclusions of our analysis.

experience less size growth. The resulting abundance increases by less than $1 \%$ compared with the fiducial abundance, but the size distribution is slightly steeper than observed at $n(r) \propto r^{-3.5}$. However, if we force the halos to have an outer slope of 3 at infall, the UDG abundance decreases by $12 \%$ and the size distribution steepens to $n(r) \propto r^{-4.3}$. Alternatively, many authors suggest that a more isothermal core is appropriate (e.g. Oh et al. 2015), which transitions more sharply from the outer slope of 3 to a central core than Eqn. 6. If our cored systems are modeled as a psudo-isothermal profile:

$$
\rho(r)=\frac{\rho_{s}}{\left(1+\left(\frac{r}{r_{s}}\right)^{2}\right)^{1.5}},
$$

matched to the $V_{\max }$ and $r_{\max }$ of the subhalo at infall, we observe a similar effect as the Di Cintio et al. (2014b) halos. More mass in the halo outskirts results in increased stripping, but increased $\gamma$ values (because of the lower core density) results in fewer large UDGs. Additionally, the lower $M_{\mathrm{vir}}$ inf values of these halos (at fixed $V_{\max }$ and $r_{\max }$ ), results in a slightly lower UDG abundance. The abundance decreases by $26 \%$ and the size distribution steepens to $n \propto r^{-4.1}$. Altogether, the halo occupation, stellar population, and environmental dependence predicted by our model are independent of the exact implementation of the cored profile, and even in extreme cases, the abundance produced by this analysis predicts a substantial UDG population.

Our prescription for dealing with tidal heating in baryondominated systems is designed to reproduce the results from Errani et al. (2018) for dark matter-dominated systems and limit the effects of tidal heating in baryon-dominated systems. Overall, this prescription has the desired effect: although some baryon-dominated systems end up as UDGs, the majority are dark-matter dominated (only $5 \%$ of systems within $10^{7}<M_{* \text {, inf }} / M_{\odot}<10^{8.5}$ have $\delta_{1 / 2}$ values greater than 1 , and $36 \%$ have $\delta_{1 / 2}$ values less than 0.1 , see Fig. A2, A3). While the adoption of an intermediate $\gamma$ is an important consideration for our full model, tidal heating of only dark

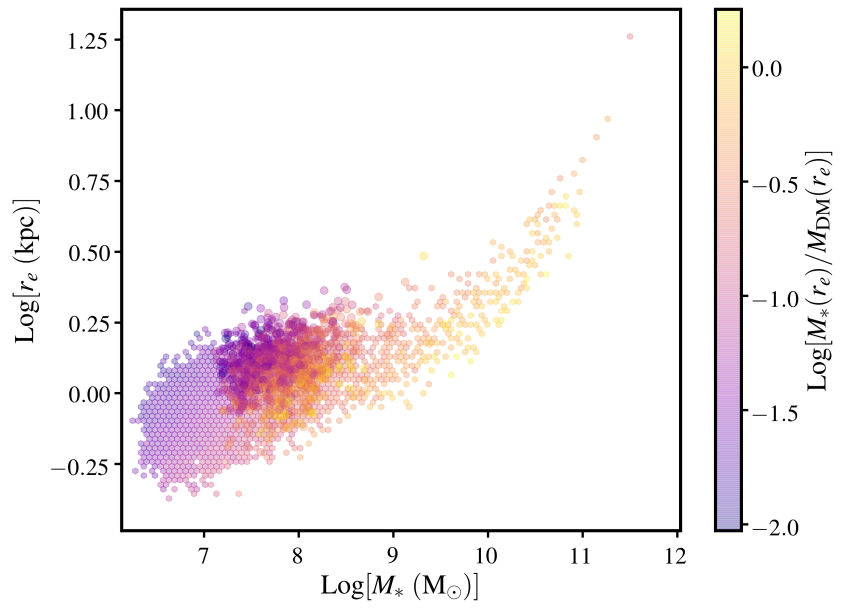

Figure A3. The infall size-mass relation, color coded by the stellar mass within $r_{e}$, with systems that end up as UDGs highlighted. Although systems with $\delta_{1 / 2}$ as high as 3 can become UDGs, the vast majority of systems that become UDGs are mildly dark-matter dominated.

matter-dominated systems in cored profiles is able to produce a reproduce many of the observed UDG properties. Although our analysis is sensitive to the value of $\delta_{1 / 2}$ for which $\gamma$ begins to change, our results do not change significantly if systems with $\delta_{1 / 2}$ above 0.5 are assigned higher $\delta_{1 / 2}$ values. Similarly, as less than $4 \%$ of systems have $\delta_{1 / 2}$ between 1 and 2 , our analysis is not sensitive to exactly how $\gamma$ depends on $\delta_{1 / 2}$ within that range (see Fig. A2). Lastly, given evidence that dwarf galaxies of all morphologies have flat stellar profiles (Faber \& Lin 1983; van Dokkum et al. 2015a; Yagi et al. 2016), the inner slope of the baryonic component is likely 0 , so $\gamma=0$ may be appropriate even in baryon-dominated cases.

Finally, we emphasize that the dark-matter halos hosting UDGs are not drawn from a particularly biased distribution. Figure A4 show both the stellar-mass-halo-mass relation and $V_{\max }-$ $r_{\max }$ relations of field halos in Illustris-dark, with systems that become UDGs highlighted. Although the systems that become UDGs tend to be larger at infall (Fig. A1, A3), the halos hosting these systems are not particularly special.

\section{APPENDIX B: UDG PROPERTIES ASSUMING AN EVOLVING SIZE-MASS RELATION}

To reproduce the observed UDG abundance, we postulate a sizemass relation that evolves weakly with redshift, following $(1+$ $z)^{-0.6}$. Here, we discuss the properties of UDGs produced in this scenario. First, although the UDG size distribution is slightly steeper than our fiducial model (see Fig. B1), it still produces a large population of large UDGs, with $2 \%$ of UDGs greater than $3 \mathrm{kpc}$. Similarly, we find that the mass distribution and velocity dispersions are not significantly changed from the non-evolving case. Lastly, while the evolving case results in younger UDGs overall, the trend between UDG age and size persists. 

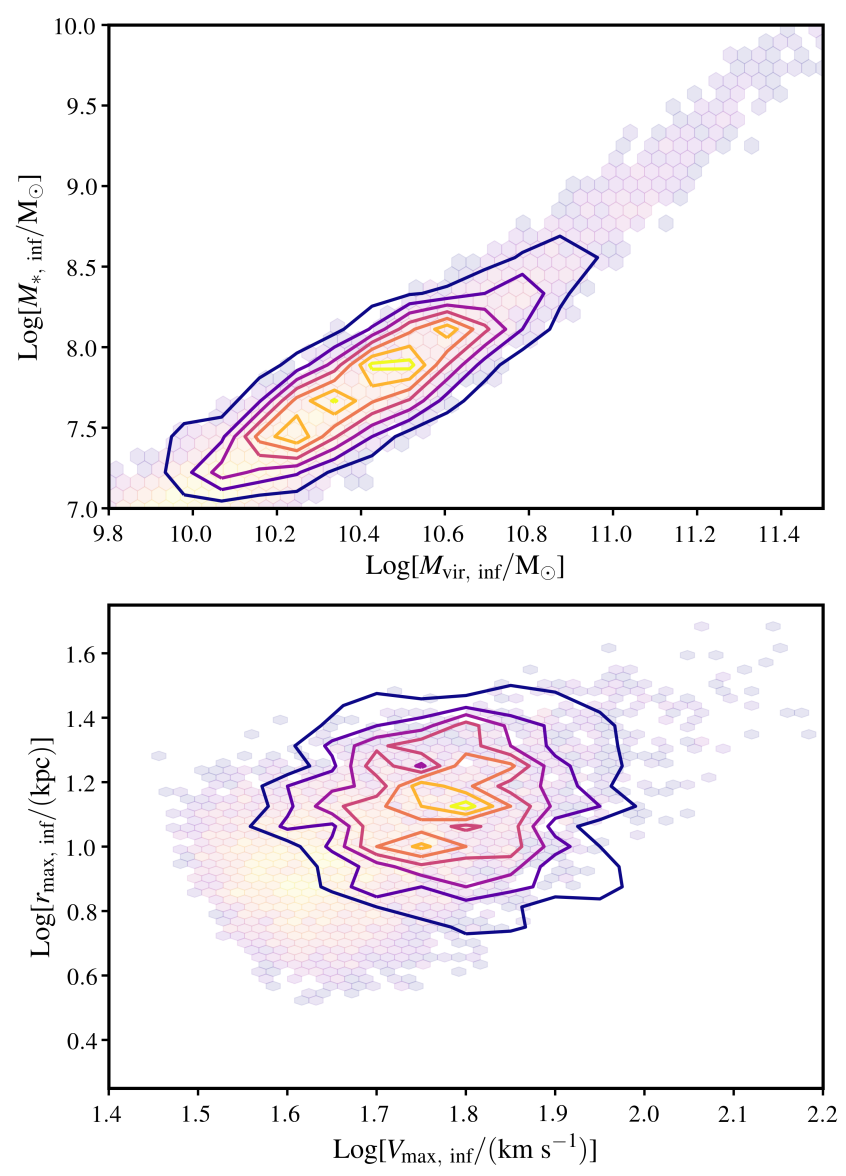

Figure A4. The infall abundance matching relation (top) and $V_{\max }-r_{\max }$ relation (bottom) color-coded by relative abundance in Illustris-dark, with systems that end up as UDGs identified as contours. In our scenario, halos hosting UDGs are cosmologically representative at infall.

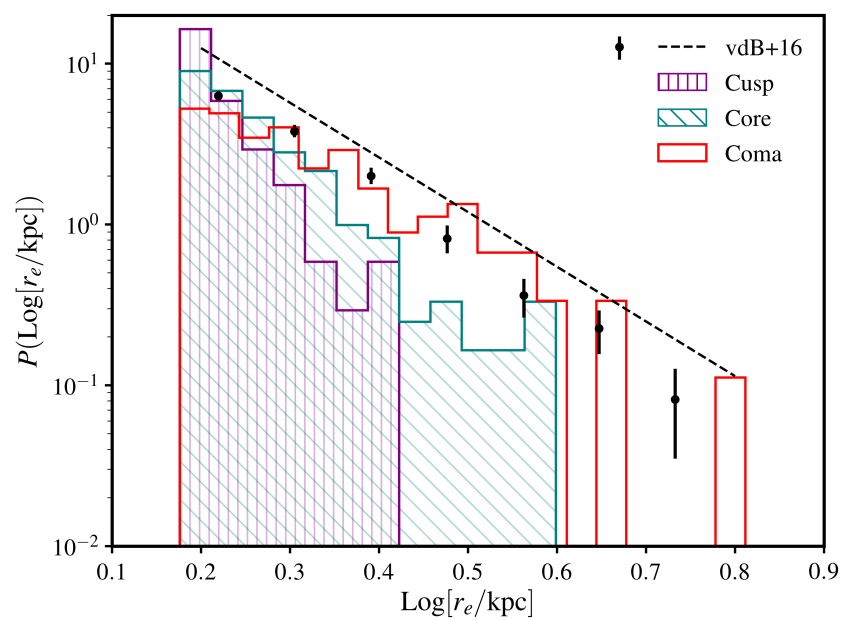

Figure B1. The size-distribution of UDGs produced through tidal stripping and heating, assuming a weakly evolving size-mass relation. Although this weak evolution decreases the abundance of the largest UDGs, the distribution is largely unchanged. 